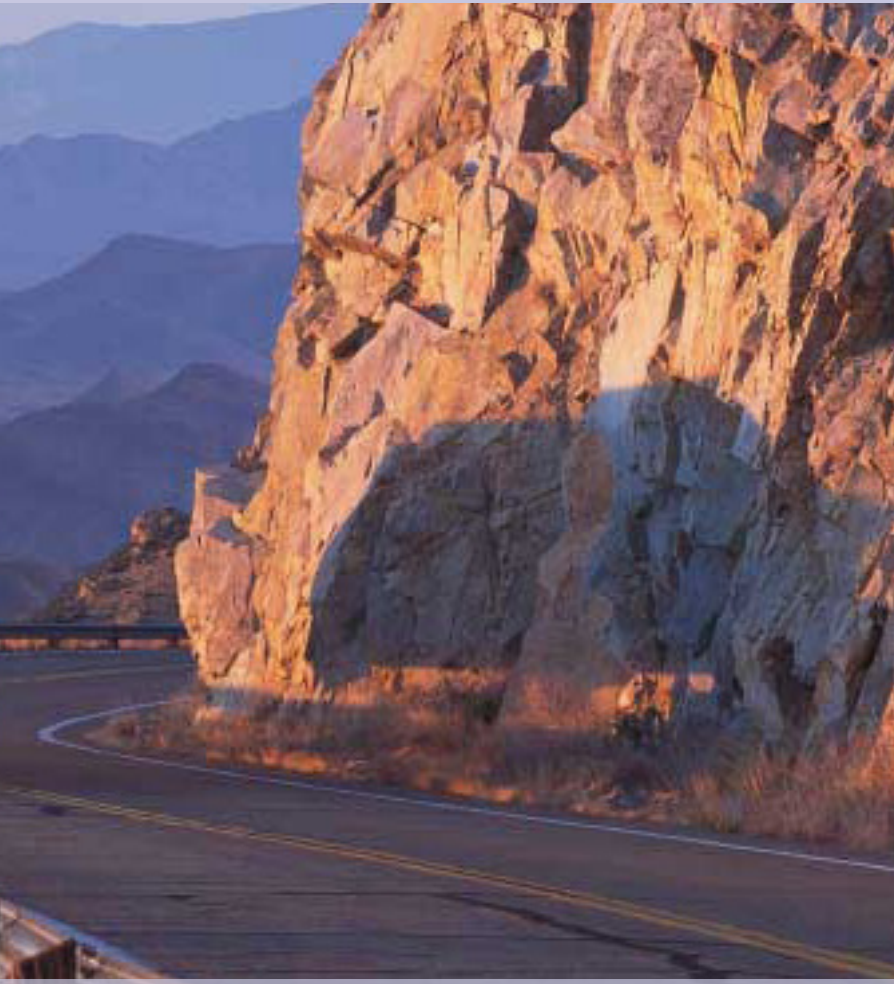

Agricultural Refrigerated

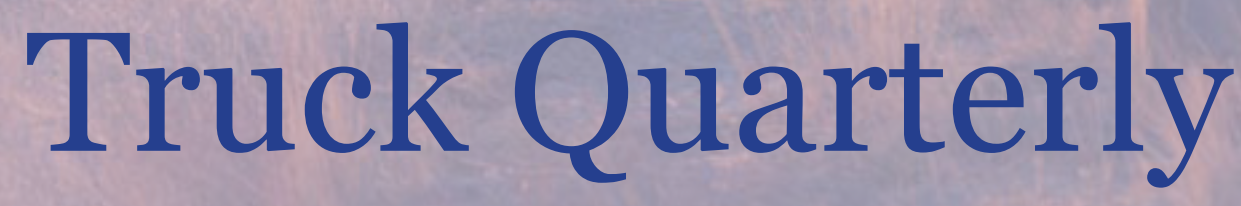

a quarterly publication of the Agricultural Marketing Service

\title{
CONTENTS
}

Quarterly Overview

.2

National Summary 3

Truck Rates. 3

Truck Rates for Selected Routes.... 4

U.S. Diesel Fuel Prices 5

Relationship Between Diesel Fuel and Truck Rates.
Quarterly Truck Availability. 8

Reported U.S. Shipments 10

Reported Shipments by Selected Commodities. 11

Regional Markets .. 12

California 12

U.S.-Mexico Border
PNW 19

Arizona 22

Florida 25

Terms and References 28 Contact Information 29 


\section{QuARTERLY OVERVIEW}

Fruit and Vegetable Shipments

During fourth quarter 2019, reported U.S. truck shipments of fresh produce were 8.73 million tons, 1 percent higher than the previous quarter and 3 percent higher than the same quarter last year.

Also, in the fourth quarter, shipments from California were higher than those from any other origin, totaling 2.44 million tons and accounting for 28 percent of the total reported shipments of fresh fruits and vegetables. Shipments from the Mexico border totaled 2.35 million tons, representing 27 percent of the total. Movements from the Pacific Northwest totaled 1.87 million tons, representing 21 percent of the reported total.

These top five commodities accounted for 38 percent of the reported truck movements during fourth quarter 2019:

Potatoes (12 percent)

Apples (10 percent)

Onions, dry (6 percent)

Oranges (6 percent)

Grapes (4 percent)

\section{Truck Rates}

The table below provides a snapshot of quarterly truck rates for U.S. produce shipments over four mileage categories-0-500; 501-1,500; 1,501-2,500; and 2,500+ miles. Please note the U.S. average truck rates provided below were calculated using weighted regional rates and volumes.

Average U.S. Truck Rates for Selected Routes between 501 and 1500 miles (\$/Mile)

\begin{tabular}{|c|c|c|c|c|}
\hline & 0-500 miles & 501-1,500 miles & $1,501-2,500$ miles & $2,501+$ miles \\
\hline Q4 2018 & 3.35 & 2.84 & 2.67 & 1.54 \\
\hline Q1 2019 & 2.90 & 2.59 & 2.46 & 1.30 \\
\hline Q2 2019 & 2.87 & 2.60 & 2.13 & 1.24 \\
\hline Q3 2019 & 4.94 & 2.47 & 2.30 & 1.35 \\
\hline Q4 2019 & 3.21 & 2.52 & 2.29 & 1.36 \\
\hline $\begin{array}{l}\text { Q4 Change from } \\
\text { Previous Quarter }\end{array}$ & $-35 \%$ & $2 \%$ & $-1 \%$ & $1 \%$ \\
\hline $\begin{array}{r}\text { Q4 Change from Same } \\
\text { Quarter Last Year }\end{array}$ & $-4 \%$ & $-11 \%$ & $-14 \%$ & $-12 \%$ \\
\hline
\end{tabular}

Source: AMS Transportation Services Division analysis of AMS Specialty Crops Program Market News data.

Note: Table values may not conform exactly due to rounding.

\section{Diesel Fuel}

During fourth quarter 2019, the U.S. diesel fuel price averaged $\$ 3.06$ per gallon-1 percent higher than the previous quarter but 6 percent lower than the same quarter last year. 


\section{NATIONAL SUMmARY}

\section{Truck Rates}

Figure 1: Average Truck Rates for Selected Routes (\$/Mile)

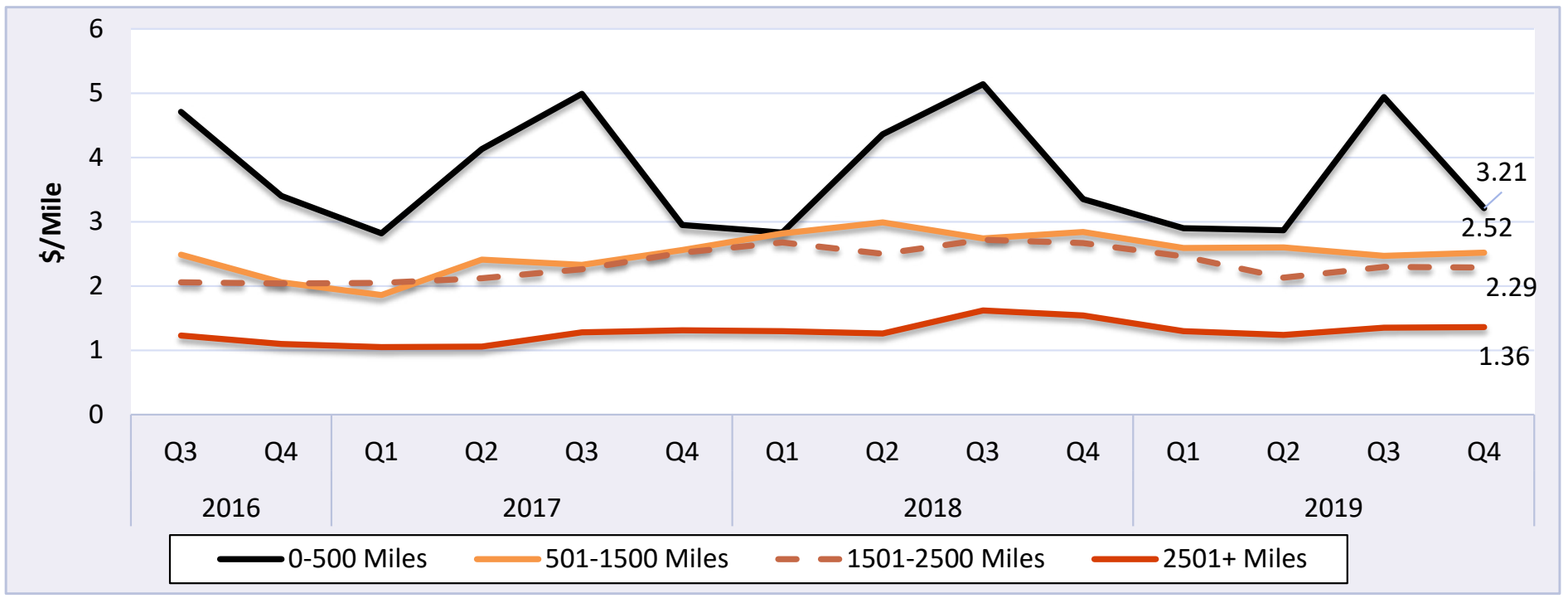

Source: AMS Transportation Services Division analysis of AMS Specialty Crops Program Market News data

Table 1: Average U.S. Truck Rates for Selected Routes between 501 and 1500 miles (\$/Mile)

\begin{tabular}{|c|c|c|c|c|c|}
\hline Year & 1st Quarter & 2nd Quarter & 3rd Quarter & 4th Quarter & Annual \\
\hline $\mathbf{2 0 1 9}$ & 2.59 & 2.60 & 2.47 & 2.52 & 2.54 \\
\hline $\mathbf{2 0 1 8}$ & 2.82 & 2.99 & 2.74 & 2.84 & 2.85 \\
\hline $\mathbf{2 0 1 7}$ & 1.86 & 2.41 & 2.33 & 2.56 & 2.29 \\
\hline $\mathbf{2 0 1 6}$ & 2.22 & 2.37 & 2.49 & 2.06 & 2.28 \\
\hline $\mathbf{2 0 1 5}$ & 2.47 & 2.63 & 2.59 & 2.36 & 2.51 \\
\hline $\mathbf{2 0 1 4}$ & 2.32 & 2.67 & 2.64 & 2.49 & 2.53 \\
\hline $\mathbf{2 0 1 3}$ & 2.24 & 2.60 & 2.62 & 2.27 & 2.43 \\
\hline $\mathbf{2 0 1 2}$ & 2.10 & 2.54 & 2.45 & 2.29 & 2.35 \\
\hline $\mathbf{2 0 1 1}$ & 2.02 & 2.60 & 2.77 & 2.26 & 2.41 \\
\hline $\mathbf{2 0 1 0}$ & 1.82 & 2.21 & 2.33 & 1.94 & 2.08 \\
\hline $\mathbf{2 0 0 9}$ & 1.85 & 1.99 & 2.02 & 1.86 & 1.93 \\
\hline $\mathbf{2 0 0 8}$ & 2.02 & 2.56 & 2.77 & 2.24 & 2.40 \\
\hline $\mathbf{2 0 0 7}$ & 1.89 & 2.23 & 2.25 & 2.03 & 2.10 \\
\hline
\end{tabular}

Source: AMS Transportation Services Division analysis of AMS Specialty Crops Program Market News data. Note: Table values may not conform exactly due to rounding. 
Table 2: Quarterly Rates for Key Origins by Month; 501-1500 miles (\$/Mile)

\begin{tabular}{|r|c|c|c|c|c|c|}
\hline \multirow{2}{*}{ Origin } & \multicolumn{3}{|c|}{ 4th Quarter, 2019 } & \multicolumn{3}{c|}{ 3rd Quarter, 2019 } \\
\cline { 2 - 7 } & October & November & December & July & August & September \\
\hline Arizona & $\mathrm{n} / \mathrm{a}$ & 3.30 & 2.85 & 3.05 & $\mathrm{n} / \mathrm{a}$ & $\mathrm{n} / \mathrm{a}$ \\
\hline Arizona-Mexico & 1.89 & 2.14 & 2.43 & 2.52 & $\mathrm{n} / \mathrm{a}$ & $\mathrm{n} / \mathrm{a}$ \\
\hline California & 2.93 & 3.05 & 3.07 & 2.97 & 2.95 & 2.81 \\
\hline Florida & 1.81 & 1.66 & 2.29 & 2.79 & $\mathrm{n} / \mathrm{a}$ & $\mathrm{n} / \mathrm{a}$ \\
\hline Great Lakes & 3.55 & 3.47 & 3.48 & 3.40 & 3.48 & 3.60 \\
\hline New York & 2.05 & 2.05 & 2.91 & 2.76 & $\mathrm{n} / \mathrm{a}$ & 2.05 \\
\hline Other & 2.25 & 2.50 & 2.66 & 2.44 & 2.37 & 2.28 \\
\hline PNW & 2.04 & 2.18 & 2.31 & 1.90 & 1.87 & 1.98 \\
\hline Southeast & 6.66 & 3.91 & 3.89 & 4.02 & 4.83 & 5.55 \\
\hline Texas & $\mathrm{n} / \mathrm{a}$ & $\mathrm{n} / \mathrm{a}$ & $\mathrm{n} / \mathrm{a}$ & 2.18 & 2.20 & 2.18 \\
\hline Texas-Mexico & 2.01 & 2.08 & 2.56 & 2.05 & 2.07 & 2.00 \\
\hline
\end{tabular}

Source: AMS Transportation Services Division analysis of AMS Specialty Crops Program Market News data.

Note: "n/a" indicates rates not available.

Note: The rates for 8 long-haul fruit and vegetable truck corridors are included in the national rate, weighted by commodity and origin volume.

\section{Truck Rates for Selected Routes}

Table 3: Origin-Destination Truck Rates for Selected Routes, 4th Quarter 2019 (\$/Mile) Destination

\begin{tabular}{|c|c|c|c|c|c|c|c|c|c|c|}
\hline \multirow[b]{2}{*}{ U.S. Origin } & \multicolumn{10}{|c|}{ Destination } \\
\hline & Atlanta & Baltimore & Boston & Chicago & Dallas & $\begin{array}{c}\text { Los } \\
\text { Angeles }\end{array}$ & Miami & $\begin{array}{l}\text { New } \\
\text { York }\end{array}$ & Philadelphia & Seattle \\
\hline Arizona & 2.55 & 2.53 & 2.49 & 2.45 & 2.98 & $\mathrm{n} / \mathrm{a}$ & 2.58 & 2.53 & 2.52 & $\mathrm{n} / \mathrm{a}$ \\
\hline $\begin{array}{l}\text { Arizona- } \\
\text { Mexico }\end{array}$ & 2.46 & 2.47 & 2.54 & 2.11 & 2.39 & 2.07 & 2.59 & 2.29 & 2.34 & $\mathrm{n} / \mathrm{a}$ \\
\hline California & 2.38 & 2.38 & 2.35 & 2.31 & 2.47 & $\mathrm{n} / \mathrm{a}$ & 2.38 & 2.41 & 2.36 & 3.27 \\
\hline Florida & 2.56 & 1.89 & 1.90 & 1.62 & $\mathrm{n} / \mathrm{a}$ & $\mathrm{n} / \mathrm{a}$ & $\mathrm{n} / \mathrm{a}$ & 2.04 & 1.89 & $\mathrm{n} / \mathrm{a}$ \\
\hline $\begin{array}{l}\text { Great } \\
\text { Lakes }\end{array}$ & 3.33 & 3.88 & 3.24 & 4.26 & 2.77 & $\mathrm{n} / \mathrm{a}$ & 2.79 & 3.93 & 3.65 & $\mathrm{n} / \mathrm{a}$ \\
\hline New York & 2.40 & 5.05 & 10.78 & $\mathrm{n} / \mathrm{a}$ & $\mathrm{n} / \mathrm{a}$ & $\mathrm{n} / \mathrm{a}$ & 2.38 & 11.67 & 6.96 & $\mathrm{n} / \mathrm{a}$ \\
\hline Other & 3.03 & 2.80 & 2.73 & 2.16 & 3.40 & 1.97 & 2.24 & 2.72 & 2.63 & $\mathrm{n} / \mathrm{a}$ \\
\hline PNW & 2.35 & 2.35 & 2.26 & 2.30 & 2.25 & 2.18 & 2.29 & 2.36 & 2.32 & 7.86 \\
\hline Southeast & 6.25 & 9.25 & 5.48 & 3.53 & $\mathrm{n} / \mathrm{a}$ & $\mathrm{n} / \mathrm{a}$ & 4.61 & 6.96 & 7.83 & $\mathrm{n} / \mathrm{a}$ \\
\hline $\begin{array}{l}\text { Texas- } \\
\text { Mexico }\end{array}$ & 2.31 & 2.36 & 2.31 & 2.15 & 2.89 & 1.72 & 2.22 & 2.40 & 2.30 & 2.11 \\
\hline
\end{tabular}

Source: AMS Transportation Services Division analysis of AMS Specialty Crops Program Market News data. 
Table 4: Origin-Destination Truck Rates for Selected Routes, 4th Quarter 2019 (\$/Truck)

\begin{tabular}{|c|c|c|c|c|c|c|c|c|c|c|}
\hline \multirow[b]{2}{*}{ U.S. Origin } & \multicolumn{10}{|c|}{ Destination } \\
\hline & Atlanta & Baltimore & Boston & Chicago & Dallas & $\begin{array}{c}\text { Los } \\
\text { Angeles }\end{array}$ & Miami & $\begin{array}{l}\text { New } \\
\text { York }\end{array}$ & Philadelphia & Seattle \\
\hline Arizona & 4,879 & 6,321 & 7,043 & 4,579 & 3,650 & $\mathrm{n} / \mathrm{a}$ & 6,600 & 6,396 & 6,213 & $\mathrm{n} / \mathrm{a}$ \\
\hline $\begin{array}{l}\text { Arizona- } \\
\text { Mexico }\end{array}$ & 4,435 & 5,807 & 6,850 & 3,800 & 2,345 & 1,158 & 5,883 & 5,723 & 5,605 & $\mathrm{n} / \mathrm{a}$ \\
\hline California & 5,351 & 6,507 & 7,201 & 4,877 & 3,717 & $\mathrm{n} / \mathrm{a}$ & 6,769 & 6,850 & 6,584 & 3,312 \\
\hline Florida & 959 & 1,881 & 2,644 & 1,736 & $\mathrm{n} / \mathrm{a}$ & $\mathrm{n} / \mathrm{a}$ & $\mathrm{n} / \mathrm{a}$ & 2,394 & 2,063 & $\mathrm{n} / \mathrm{a}$ \\
\hline $\begin{array}{l}\text { Great } \\
\text { Lakes }\end{array}$ & 3,317 & 3,523 & 4,270 & 1,401 & 3,073 & $\mathrm{n} / \mathrm{a}$ & 4,982 & 3,919 & 3,475 & $\mathrm{n} / \mathrm{a}$ \\
\hline New York & 2,400 & 1,667 & 1,833 & $\mathrm{n} / \mathrm{a}$ & $\mathrm{n} / \mathrm{a}$ & $\mathrm{n} / \mathrm{a}$ & 3,450 & 1,750 & 1,600 & $\mathrm{n} / \mathrm{a}$ \\
\hline Other & 2,013 & 3,600 & 3,572 & 2,024 & 1,787 & 1,828 & 4,587 & 3,700 & 3,558 & $\mathrm{n} / \mathrm{a}$ \\
\hline PNW & 5,408 & 5,737 & 6,187 & 4,078 & 4,082 & 2,156 & 6,512 & 5,986 & 5,802 & 1,100 \\
\hline Southeast & 2,500 & 3,053 & 4,053 & 3,000 & $\mathrm{n} / \mathrm{a}$ & $\mathrm{n} / \mathrm{a}$ & 3,553 & 3,618 & 3,368 & $\mathrm{n} / \mathrm{a}$ \\
\hline $\begin{array}{r}\text { Texas- } \\
\text { Mexico }\end{array}$ & 2,654 & 4,218 & 5,079 & 3,079 & 1,443 & 2,750 & 3,393 & 4,804 & 4,371 & 5,071 \\
\hline
\end{tabular}

Source: AMS Transportation Services Division analysis of AMS Specialty Crops Program Market News data.

Note: "n/a" indicates rates not available

\section{U.S. Diesel Fuel Prices}

The diesel fuel price provides a proxy for trends in U.S. truck rates. Diesel fuel is a significant component underlying truck rates.

Figure 2: U.S. Average On-Highway Diesel Fuel Prices

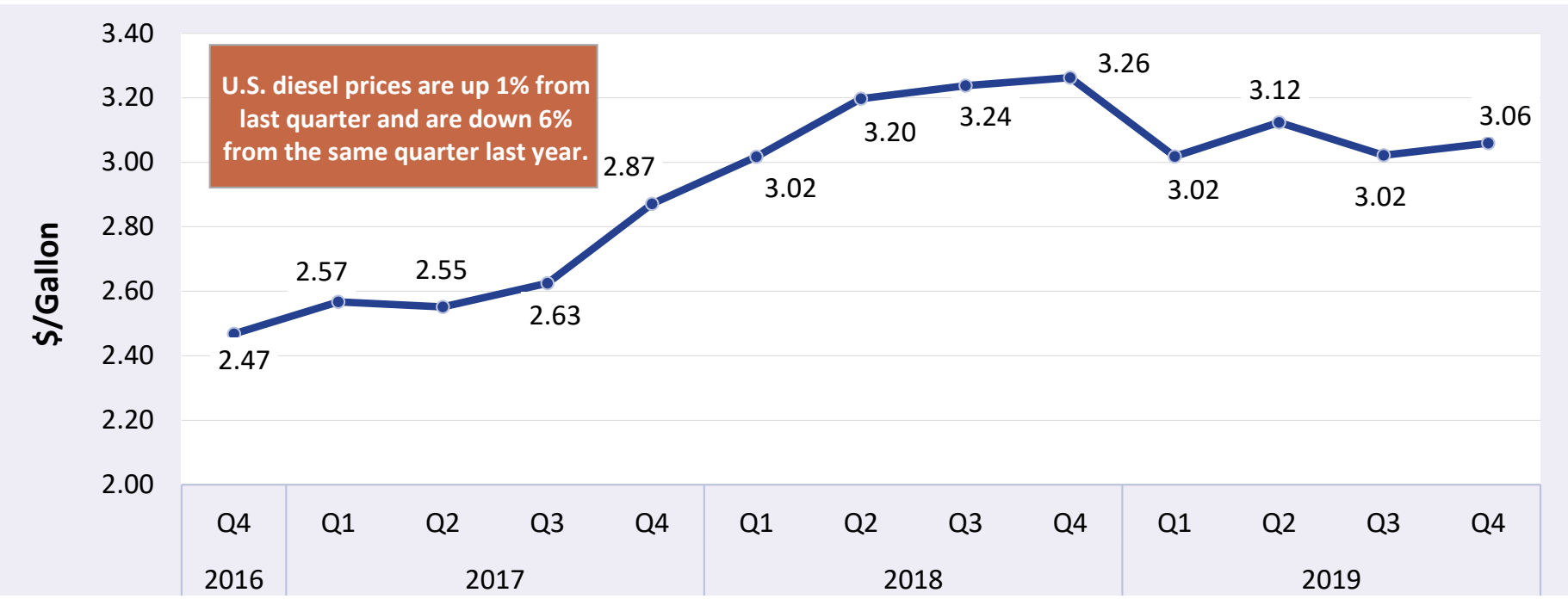

Source: AMS Transportation Services Division analysis of Energy Information Administration/U.S. Department of Energy data. 
Table 5: Average Diesel Fuel Prices (All Types)

\begin{tabular}{|r|c|c|c|}
\hline \multicolumn{1}{|c|}{ Region } & $\begin{array}{c}\text { 4th Quarter 2019 Price } \\
\text { \$/Gallon }\end{array}$ & $\begin{array}{c}\text { Change From Last } \\
\text { Quarter }\end{array}$ & $\begin{array}{c}\text { Change From Same } \\
\text { Quarter Last Year }\end{array}$ \\
\hline East Coast & 3.05 & $0 \%$ & $-7 \%$ \\
\hline California & 3.96 & $1 \%$ & $-1 \%$ \\
\hline New England & 3.06 & $-1 \%$ & $-8 \%$ \\
\hline Lower Atlantic & 3.24 & $1 \%$ & $-6 \%$ \\
\hline Gulf Coast & 2.92 & $0 \%$ & $-7 \%$ \\
\hline Midwest & 2.97 & $0 \%$ & $-8 \%$ \\
\hline Rocky Mountain & 3.14 & $1 \%$ & $-7 \%$ \\
\hline West Coast Except California & 3.33 & $6 \%$ & $-5 \%$ \\
\hline U.S. & 3.06 & $5 \%$ & $-4 \%$ \\
\hline
\end{tabular}

Source: AMS Transportation Services Division analysis of Energy Information Administration/U.S. Department of Energy data.

Note: Table values may not conform exactly due to rounding.

\section{Relationship Between Diesel Fuel and Truck Rates}

The diesel fuel price provides a proxy for trends in U.S. truck rates. Diesel fuel is a significant expense for fruit and vegetable movements.

Figure 3: U.S. Average On-Highway Diesel Fuel Prices and Truck Rates

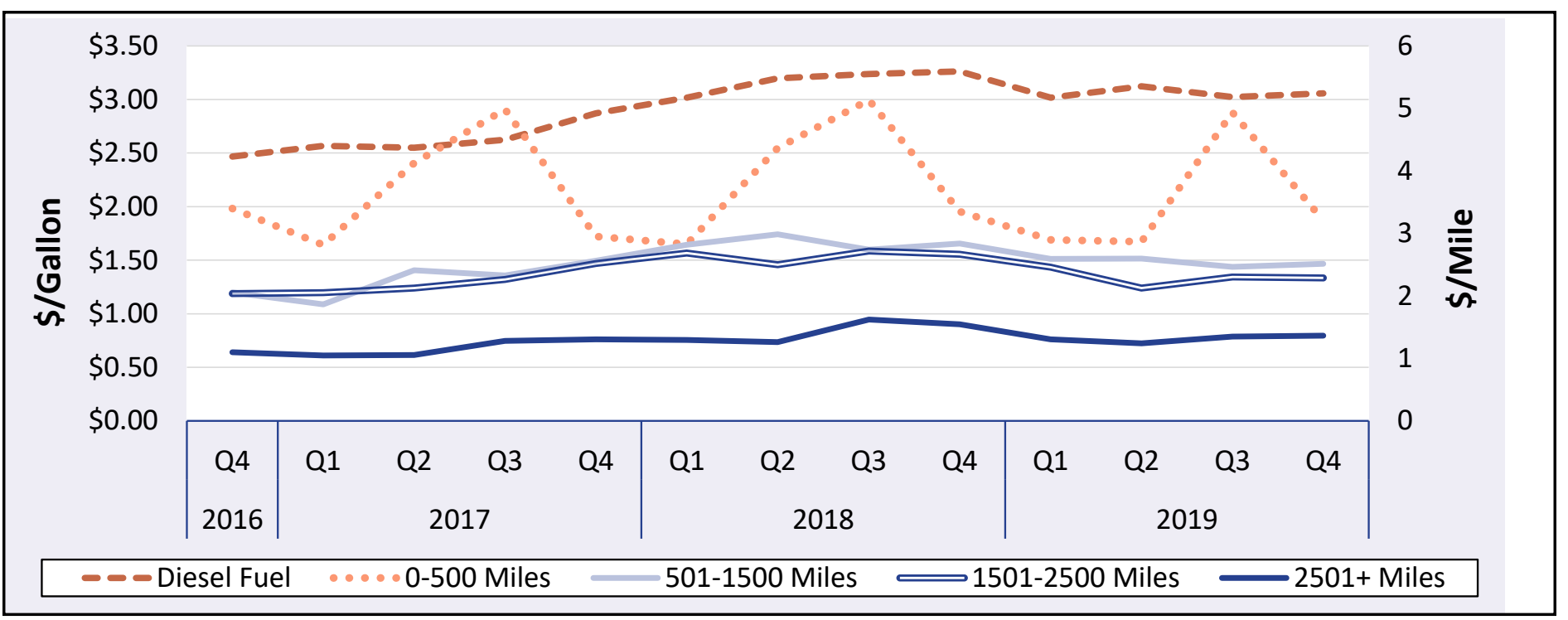

Sources: AMS Transportation Services Division analysis of AMS Specialty Crops Program Market News data and Energy Information Administration/ U.S. Department of Energy data. 
Table 6: Average Diesel Fuel Prices and Truck Rates

\begin{tabular}{|c|c|c|c|c|c|c|c|}
\hline \multirow{3}{*}{ Year } & \multirow{3}{*}{ Quarter } & \multirow{3}{*}{$\begin{array}{l}\text { Diesel Fuel } \\
\text { (\$/gallon) }\end{array}$} & \multirow{3}{*}{$\begin{array}{c}\text { Truck Rates } \\
\text { (\$/mile) } \\
\text { 501-1500 } \\
\text { miles }\end{array}$} & \multicolumn{4}{|c|}{$\%$ Change From } \\
\hline & & & & \multicolumn{2}{|c|}{ Last Quarter } & \multicolumn{2}{|c|}{ Last Year } \\
\hline & & & & Diesel & Truck & Diesel & Truck \\
\hline 2016 & Q4 & 2.47 & 2.06 & $4 \%$ & $-17 \%$ & $1 \%$ & $-13 \%$ \\
\hline \multirow[t]{4}{*}{2017} & Q1 & 2.57 & 1.86 & $4 \%$ & $-9 \%$ & $24 \%$ & $-16 \%$ \\
\hline & Q2 & 2.55 & 2.41 & $-1 \%$ & $29 \%$ & $11 \%$ & $2 \%$ \\
\hline & Q3 & 2.63 & 2.33 & $3 \%$ & $-4 \%$ & $10 \%$ & $-7 \%$ \\
\hline & Q4 & 2.87 & 2.56 & $9 \%$ & $10 \%$ & $16 \%$ & $25 \%$ \\
\hline \multirow[t]{4}{*}{2018} & Q1 & 3.02 & 2.82 & $5 \%$ & $10 \%$ & $18 \%$ & $51 \%$ \\
\hline & Q2 & 3.20 & 2.99 & $6 \%$ & $6 \%$ & $25 \%$ & $24 \%$ \\
\hline & Q3 & 3.24 & 2.74 & $1 \%$ & $-8 \%$ & $23 \%$ & $18 \%$ \\
\hline & Q4 & 3.26 & 2.84 & $1 \%$ & $4 \%$ & $14 \%$ & $11 \%$ \\
\hline \multirow[t]{4}{*}{2019} & Q1 & 3.02 & 2.59 & $-8 \%$ & $-9 \%$ & $0 \%$ & $-8 \%$ \\
\hline & Q2 & 3.12 & 2.60 & $4 \%$ & $0 \%$ & $-2 \%$ & $-13 \%$ \\
\hline & Q3 & 3.02 & 2.47 & $-3 \%$ & $-5 \%$ & $-7 \%$ & $-10 \%$ \\
\hline & Q4 & 3.06 & 2.52 & $1 \%$ & $2 \%$ & $-6 \%$ & $-11 \%$ \\
\hline
\end{tabular}

Sources: AMS Transportation Services Division analysis of AMS Specialty Crops Program Market News data and Energy Information Administration/U.S. Department of Energy data.

Note: Table values may not conform exactly due to rounding. 


\section{Quarterly Truck Availability}

Table 7: U.S. Fresh Fruit and Vegetable Truck Availability

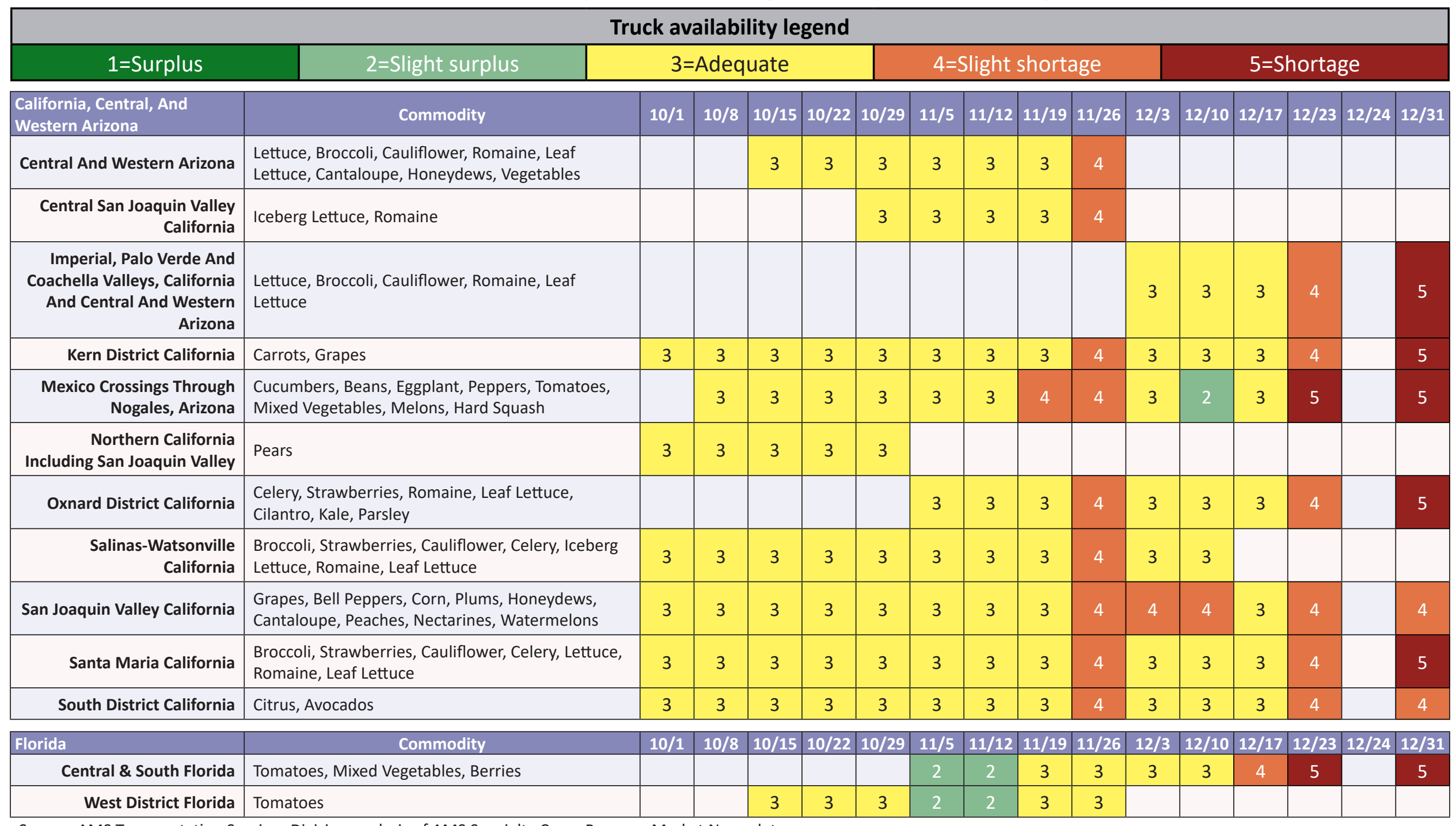

Source: AMS Transportation Services Division analysis of AMS Specialty Crops Program Market News data

Note: Empty cells were not reported. 
Table 7, continued: U.S. Fresh Fruit and Vegetable Truck Availability

\begin{tabular}{|c|c|c|c|c|c|c|c|c|c|c|c|c|c|c|c|c|}
\hline \multicolumn{17}{|c|}{ Truck availability legend } \\
\hline 1=Surplus & 2=Slight surplus & \multicolumn{4}{|c|}{ 3=Adequate } & \multicolumn{5}{|c|}{ 4=Slight shortage } & \multicolumn{6}{|c|}{ 5=Shortage } \\
\hline Great Lakes (MI \& WI) & Commodity & $10 / 1$ & $10 / 8$ & $10 / 15$ & $10 / 22$ & $10 / 29$ & $11 / 5$ & $11 / 12$ & $11 / 19$ & $11 / 26$ & $12 / 3$ & $12 / 10$ & $12 / 17$ & $12 / 23$ & $12 / 24$ & $12 / 31$ \\
\hline Central Wisconsin & Potatoes, Onions & 3 & 3 & 3 & 3 & 3 & 3 & 3 & 3 & 3 & 3 & 3 & 3 & 3 & 3 & 5 \\
\hline Michigan & Apples & 3 & 3 & 3 & 3 & 3 & 3 & 3 & 3 & 3 & 3 & 3 & 3 & 3 & & 3 \\
\hline U.S.-Mexico border & Commodity & $10 / 1$ & $10 / 8$ & $10 / 15$ & $10 / 22$ & $10 / 29$ & $11 / 5$ & $11 / 12$ & $11 / 19$ & $11 / 26$ & $12 / 3$ & $12 / 10$ & $12 / 17$ & $12 / 23$ & $12 / 24$ & $12 / 31$ \\
\hline Texas & $\begin{array}{l}\text { Limes, Tomatoes, Broccoli, Mixed Fruit and } \\
\text { Vegetables }\end{array}$ & 3 & 3 & 3 & 3 & 3 & 3 & 3 & 3 & 3 & 3 & 3 & 3 & 3 & & 3 \\
\hline $\begin{array}{l}\text { Pacific Northwest (ID, OR, \&, } \\
\text { WA) }\end{array}$ & Commodity & $10 / 1$ & $10 / 8$ & $10 / 15$ & $10 / 22$ & $10 / 29$ & $11 / 5$ & $11 / 12$ & $11 / 19$ & $11 / 26$ & $12 / 3$ & $12 / 10$ & $12 / 17$ & $12 / 23$ & $12 / 24$ & $12 / 31$ \\
\hline Columbia Basin Washington & Potatoes, Onions & 3 & 3 & 3 & 3 & 3 & 3 & 3 & 4 & 4 & 4 & 4 & 4 & 4 & 4 & 5 \\
\hline $\begin{array}{r}\text { Idaho And Malheur County, } \\
\text { Oregon }\end{array}$ & Onions & 3 & 3 & 3 & 3 & 3 & 3 & 3 & 4 & 4 & 4 & 3 & 4 & 4 & 4 & 4 \\
\hline $\begin{array}{l}\text { Upper Valley, Twin Falls- } \\
\text { Burley District Idaho }\end{array}$ & Potatoes & 4 & 3 & 3 & 3 & 3 & 3 & 3 & 4 & 4 & 4 & 3 & 4 & 4 & 4 & 5 \\
\hline $\begin{array}{r}\text { Yakima Valley \& Wenatchee } \\
\text { District Washington }\end{array}$ & Apples, Pears & 3 & 3 & 3 & 3 & 3 & 3 & 3 & 3 & 4 & 3 & 3 & 3 & 4 & & 4 \\
\hline Southeast (GA, SC, \& NC) & Commodity & $10 / 1$ & $10 / 8$ & $10 / 15$ & $10 / 22$ & $10 / 29$ & $11 / 5$ & $11 / 12$ & $11 / 19$ & $11 / 26$ & $12 / 3$ & $12 / 10$ & $12 / 17$ & $12 / 23$ & $12 / 24$ & $12 / 31$ \\
\hline Eastern North Carolina & Sweet Potatoes & 3 & 4 & 4 & 4 & 4 & 3 & 2 & 5 & 5 & 4 & 4 & 3 & 5 & & 4 \\
\hline
\end{tabular}

Source: AMS Transportation Services Division analysis of AMS Specialty Crops Program Market News data Note: Empty cells were not reported. 


\section{Reported U.S. Shipments}

Figure 4: Reported U.S. Fruit and Vegetable Shipments (1,000 Tons)

12,000

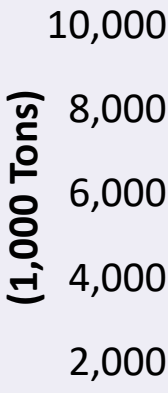

0
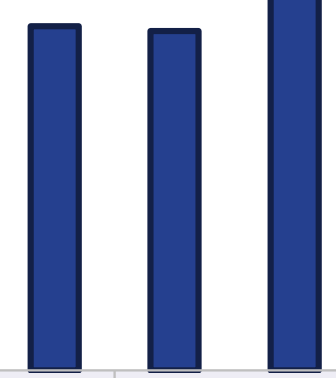

$4 Q$

2016
10

$2 \mathrm{Q}$

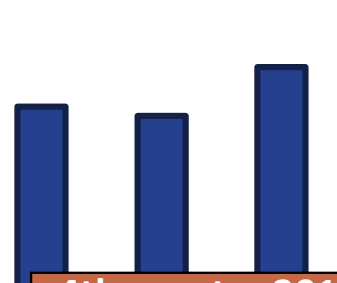

4th quarter 2019 U.S. shipments increased 1\% compared to the previous quarter, and

increased $3 \%$ compared to the same quarter

last year.

2017

2018

2019

Source: AMS Transportation Services Division analysis of AMS Specialty Crops Program Market News data

Table 8: Reported U.S. Fruit and Vegetable Shipments (1,000 Tons)

\begin{tabular}{|r|c|c|c|c|c|}
\hline \multicolumn{1}{|c|}{ Year } & 1st Quarter & 2nd Quarter & 3rd Quarter & 4th Quarter & Annual \\
\hline $\mathbf{2 0 1 9}$ & 9,381 & 10,246 & 8,687 & 8,732 & 37,046 \\
\hline $\mathbf{2 0 1 8}$ & 9,421 & 10,795 & 8,789 & 8,503 & 37,508 \\
\hline $\mathbf{2 0 1 7}$ & 8,072 & 9,642 & 8,479 & 8,267 & 34,459 \\
\hline $\mathbf{2 0 1 6}$ & 8,094 & 9,761 & 8,541 & 8,188 & 34,583 \\
\hline $\mathbf{2 0 1 5}$ & 8,118 & 9,630 & 8,324 & 7,771 & 33,842 \\
\hline $\mathbf{2 0 1 4}$ & 7,733 & 9,139 & 8,080 & 7,725 & 32,677 \\
\hline $\mathbf{2 0 1 3}$ & 7,451 & 8,972 & 7,762 & 7,527 & 31,712 \\
\hline $\mathbf{2 0 1 2}$ & 7,577 & 9,008 & 7,774 & 7,532 & 31,890 \\
\hline $\mathbf{2 0 1 1}$ & 7,007 & 8,981 & 7,887 & 7,988 & 31,863 \\
\hline $\mathbf{2 0 1 0}$ & 7,065 & 8,881 & 7,985 & 7,522 & 31,454 \\
\hline $\mathbf{2 0 0 9}$ & 7,158 & 8,728 & 7,990 & 7,270 & 31,147 \\
\hline $\mathbf{2 0 0 8}$ & 7,059 & 8,666 & 7,426 & 6,904 & 30,057 \\
\hline $\mathbf{2 0 0 7}$ & 6,959 & 8,585 & 7,475 & 7,099 & 30,118 \\
\hline $\mathbf{2 0 0 6}$ & 6,335 & 8,400 & 7,854 & 6,960 & 29,550 \\
\hline $\mathbf{2 0 0 5}$ & 6,877 & 8,324 & 7,737 & 7,387 & 30,325 \\
\hline $\mathbf{2 0 0 4}$ & 6,867 & 8,331 & 6,876 & 6,732 & 28,807 \\
\hline $\mathbf{2 0 0 3}$ & 6,824 & 8,013 & 7,043 & 6,684 & 28,564 \\
\hline
\end{tabular}

Source: AMS Transportation Services Division analysis of AMS Specialty Crops Program Market News data

Note: Table values may not conform exactly due to rounding. 


\section{Reported Shipments by Selected Commodities}

Table 9: Reported Top 10 Commodity Shipments (1,000 Tons)

\begin{tabular}{|c|c|c|c|c|c|}
\hline \multirow{2}{*}{ Commodity } & \multirow{2}{*}{$\begin{array}{c}\text { 4th Quarter } \\
2019 \\
\end{array}$} & \multirow{2}{*}{$\begin{array}{l}\text { Previous } \\
\text { Quarter }\end{array}$} & \multirow{2}{*}{$\begin{array}{c}\text { Same Quarter } \\
\text { Last Year }\end{array}$} & \multicolumn{2}{|c|}{ Current Quarter as \% change from: } \\
\hline & & & & Previous Qtr & Same Qtr Last Year \\
\hline Potatoes & 1,090 & 1,062 & 1,134 & $3 \%$ & $-4 \%$ \\
\hline Apples & 903 & 621 & 805 & $46 \%$ & $12 \%$ \\
\hline Onions Dry & 549 & 564 & 579 & $-3 \%$ & $-5 \%$ \\
\hline Oranges & 481 & 245 & 441 & $97 \%$ & $9 \%$ \\
\hline Grapes & 345 & 386 & 351 & $-11 \%$ & $-2 \%$ \\
\hline Tomatoes & 331 & 274 & 341 & $21 \%$ & $-3 \%$ \\
\hline Lettuce, Iceberg & 317 & 324 & 326 & $-2 \%$ & $-3 \%$ \\
\hline Avocados & 299 & 265 & 260 & $13 \%$ & $15 \%$ \\
\hline $\begin{array}{r}\text { Lettuce, } \\
\text { Romaine }\end{array}$ & 251 & 243 & 266 & $3 \%$ & $-6 \%$ \\
\hline Cucumbers & 247 & 159 & 254 & $56 \%$ & $-2 \%$ \\
\hline
\end{tabular}

Source: AMS Transportation Services Division analysis of AMS Specialty Crops Program Market News data Note: Table values may not conform exactly due to rounding.

Table 10: Reported Top 10 Regions (1,000 Tons)

\begin{tabular}{|r|c|c|c|}
\hline \multicolumn{1}{|c|}{ Origin } & 4th Quarter 2019 Volume & $\%$ Change from Last Quarter & $\begin{array}{c}\text { Change From Same Quarter } \\
\text { Last Year }\end{array}$ \\
\hline California & 2440 & $-28 \%$ & $5 \%$ \\
\hline Mexico & 2348 & $35 \%$ & $5 \%$ \\
\hline PNW & 1871 & $21 \%$ & $-12 \%$ \\
\hline Arizona & 422 & $1,129 \%$ & $4 \%$ \\
\hline Florida & 387 & $1,710 \%$ & $-10 \%$ \\
\hline Other & 269 & $-39 \%$ & $1 \%$ \\
\hline Colorado & 266 & $-21 \%$ & $-6 \%$ \\
\hline Southeast & 224 & $41 \%$ & $13 \%$ \\
\hline New York & 201 & $-47 \%$ & $3 \%$ \\
\hline
\end{tabular}

Source: AMS Transportation Services Division analysis of AMS Specialty Crops Program Market News data

Note: Table values may not conform exactly due to rounding. 


\section{REgional MARKETS California}

\section{Volume}

Total reported shipments of fruits and vegetables from California during fourth quarter 2019 were 2.44 million tons, a quarter-to-quarter decrease of 28 percent. The top five commodities increased 18 percent from fourth quarter 2018 (year to year). Of the top 5 commodities, grapes, lemons, and celery decreased 2, 5, and 5 percent, respectively, year-to-year. Oranges increased 11 percent year-to-year and reported clementine shipments increased significantly.

\section{Rates}

The quarterly average truck rate for shipments between 501 miles and 1,500 miles was $\$ 3.02$ per mile, a 3-percent increase quarter to quarter, but a 6-percent decrease year to year.

\section{Truck Overview}

Diesel fuel prices averaged $\$ 3.96$ per gallon, a 1-percent increase quarter to quarter but a 1-percent decrease year to year. Shippers reported adequate truck availability for most of fourth quarter 2019 except for slight shortage conditions the last week in November and shortage conditions the last 2 weeks in December.

Table 11: Reported Top Five Commodities Shipped from California (1,000 tons)

\begin{tabular}{|c|c|c|c|c|c|c|}
\hline \multirow{2}{*}{ Commodity } & \multirow{2}{*}{$\begin{array}{c}\text { 4th Quarter } \\
2019\end{array}$} & \multirow{2}{*}{$\begin{array}{c}\text { Share of } \\
\text { California } \\
\text { Total }\end{array}$} & \multirow{2}{*}{$\begin{array}{l}\text { Previous } \\
\text { Quarter }\end{array}$} & \multirow{2}{*}{$\begin{array}{c}\text { Same } \\
\text { Quarter Last } \\
\text { Year }\end{array}$} & \multicolumn{2}{|c|}{$\begin{array}{c}\text { Current Quarter as \% } \\
\text { change from: }\end{array}$} \\
\hline & & & & & Previous Qtr & $\begin{array}{l}\text { Same Qtr } \\
\text { Last Year }\end{array}$ \\
\hline Oranges & 417 & $17 \%$ & 241 & 377 & $73 \%$ & $11 \%$ \\
\hline Grapes & 345 & $14 \%$ & 369 & 351 & $-7 \%$ & $-2 \%$ \\
\hline Clementines & 231 & $9 \%$ & 0 & 28 & - & $713 \%$ \\
\hline Lemons & 204 & $8 \%$ & 86 & 214 & $139 \%$ & $-5 \%$ \\
\hline Celery & 201 & $8 \%$ & 154 & 211 & $31 \%$ & $-5 \%$ \\
\hline Top 5 Total & 1,399 & $57 \%$ & 849 & 1,182 & $65 \%$ & $18 \%$ \\
\hline California Total & 2,440 & $100 \%$ & 3,407 & 2,333 & $-28 \%$ & $5 \%$ \\
\hline
\end{tabular}

Source: AMS Transportation Services Division analysis of AMS Specialty Crops Program Market News data.

"-" indicates no reported shipments during the quarter.

Note: Table values may not conform exactly due to rounding. 
Figure 5: California Truck Rates (\$/Mile by Distance Travelled)

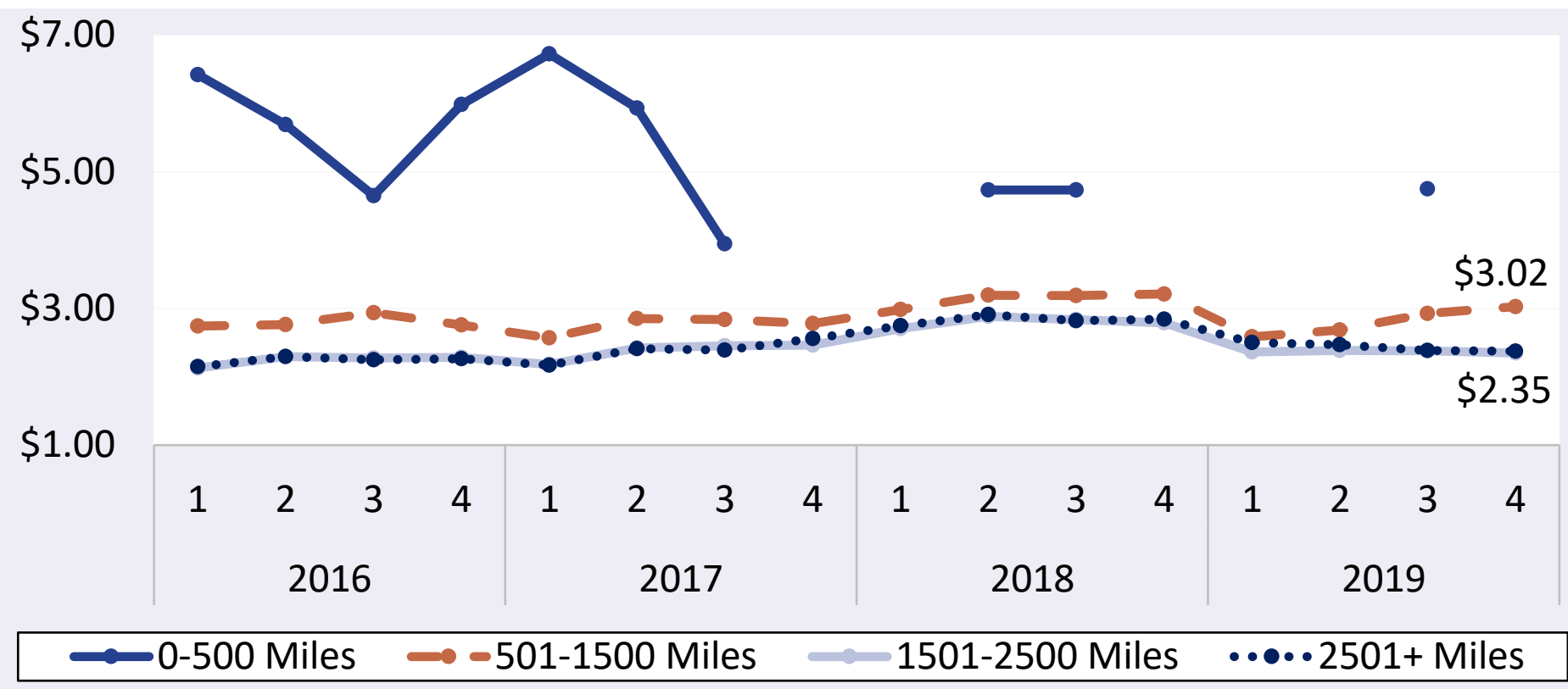

Source: AMS Transportation Services Division analysis of AMS Specialty Crops Program Market News data.

Gaps in the chart lines are the result of quarters with no reported data for the region.

Table 12: California Truck Overview (Availability Rating: 1=Surplus to 5=Shortage)

\begin{tabular}{|r|c|c|c|c|}
\hline Region/Reporting District & October & November & December & 4th Quarter \\
\hline Central San Joaquin Valley California & 3 & 3.25 & - & 3.13 \\
\hline $\begin{array}{r}\text { Imperial, Palo Verde And Coachella Valleys, } \\
\text { California And Central And Western Arizona }\end{array}$ & - & - & 3.6 & 3.6 \\
\hline Kern District California & 3 & 3.25 & 3.6 & 3.28 \\
\hline Northern California Including San Joaquin Valley & 3 & - & - & 3 \\
\hline Oxnard District California & - & 3.25 & 3.6 & 3.43 \\
\hline Salinas-Watsonville California & 3 & 3.25 & 3 & 3.08 \\
\hline San Joaquin Valley California & 3 & 3.25 & 3.8 & 3.35 \\
\hline Santa Maria California & 3 & 3.25 & 3.6 & 3.28 \\
\hline South District California & 3 & 3.25 & 3.4 & 3.22 \\
\hline Regional Average Availability & 3 & 3.25 & 3.51 & 3.25 \\
\hline Diesel Fuel Price (\$)/gallon) & 3.99 & 4.00 & 3.90 & 3.96 \\
\hline
\end{tabular}

Source: AMS Transportation Services Division analysis of AMS Specialty Crops Program Market News data.

"-" indicates no reported shipments during the quarter.

Note: Table values may not conform exactly due to rounding. 
Figure 6: Refrigerated Truck Availability Monthly Ratings for California

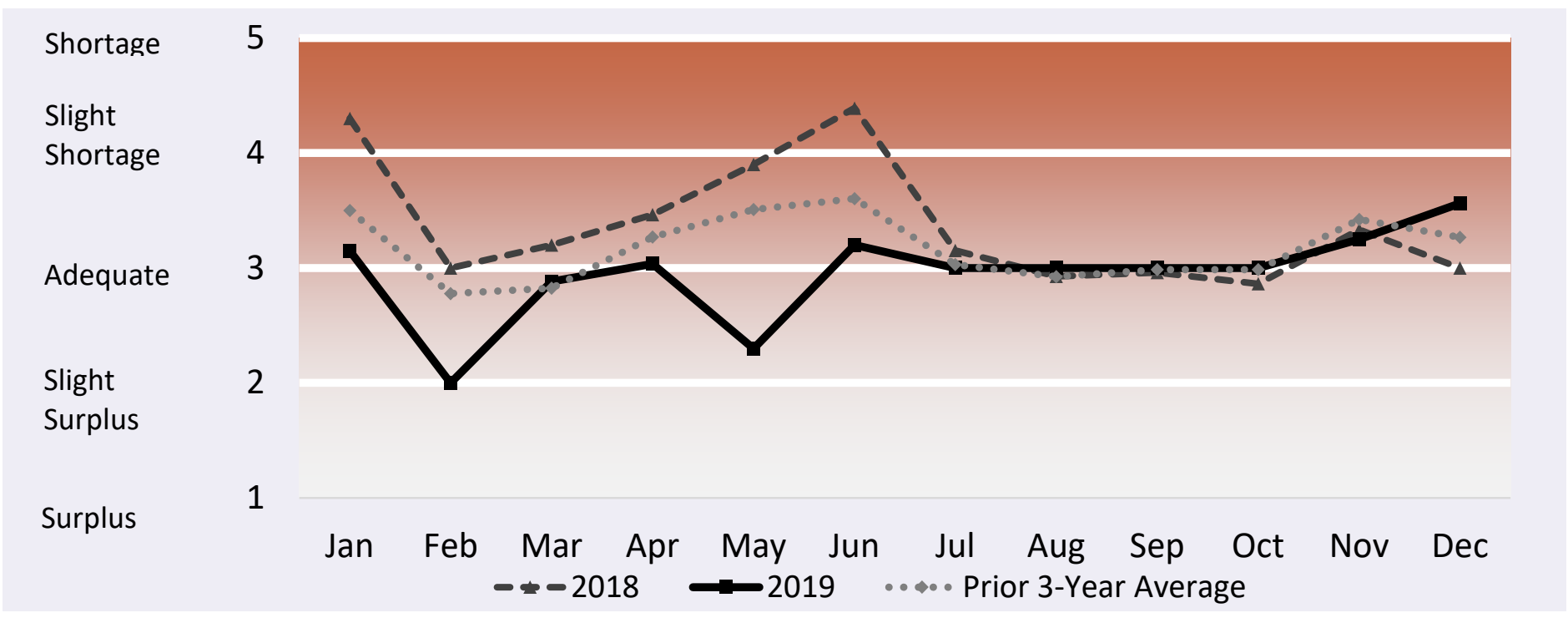

Source: AMS Transportation Services Division analysis of AMS Specialty Crops Program Market News data.

Gaps in the chart lines are the result of quarters with no reported data for the region. 


\section{U.S.-Mexico Border}

\section{Volume}

Total reported shipments of fruits and vegetables from Mexico during fourth quarter 2019 were 2.35 million tons, a 5-percent increase year to year. The sum of the top five commodities increased 2 percent year to year. Avocados saw the largest year-to-year increase at 18 percent. Also, year to year, shipments of cucumbers and peppers decreased 5 percent and 10 percent, respectively.

\section{Rates}

In fourth quarter 2019, truck rates for shipments between 501 miles and 1,500 miles from the Arizona border crossings averaged $\$ 2.21$ per mile, down 12 percent from third quarter 2019 (quarter to quarter) and down 11 percent year to year. Rates for shipments between 501 miles and 1,500 miles from the Texas border crossings averaged $\$ 2.23$ per mile, up 9 percent quarter to quarter, but down 5 percent year to year.

\section{Truck Overview}

Diesel fuel prices for border crossings from Arizona averaged \$3.33 per gallon, increasing 5 percent quarter to quarter but decreasing 4 percent year to year. Diesel fuel prices for border crossings from Texas averaged \$2.79 per gallon, a 0.2-percent increase quarter to quarter, but an 8-percent decrease year to year. Shippers reported adequate truck availability for crossing the Arizona border in October through mid-November; at the end of November, conditions changed to slight shortage. In the beginning of December, truck availability was reported as adequate, then shifted to shortage by the end of the month. At Texas border-crossing locations, shippers reported adequate truck availability throughout fourth quarter 2019.

Table 13: Reported Top Five Commodities Shipped from Mexico (1,000 tons)

\begin{tabular}{|c|c|c|c|c|c|c|}
\hline \multirow{2}{*}{ Commodity } & \multirow{2}{*}{$\begin{array}{c}\text { 4th Quarter } \\
2019\end{array}$} & \multirow{2}{*}{$\begin{array}{l}\text { Share of } \\
\text { Mexico Total }\end{array}$} & \multirow{2}{*}{$\begin{array}{l}\text { Previous } \\
\text { Quarter }\end{array}$} & \multirow{2}{*}{$\begin{array}{c}\text { Same } \\
\text { Quarter Last } \\
\text { Year }\end{array}$} & \multicolumn{2}{|c|}{$\begin{array}{c}\text { Current Quarter as \% } \\
\text { change from: }\end{array}$} \\
\hline & & & & & Previous Qtr & $\begin{array}{l}\text { Same Qtr } \\
\text { Last Year }\end{array}$ \\
\hline Avocados & 293 & $12 \%$ & 211 & 249 & $39 \%$ & $18 \%$ \\
\hline Cucumbers & 207 & $9 \%$ & 95 & 216 & $118 \%$ & $-5 \%$ \\
\hline Tomatoes & 168 & $7 \%$ & 117 & 167 & $44 \%$ & $1 \%$ \\
\hline Peppers, Other & 159 & $7 \%$ & 141 & 176 & $13 \%$ & $-10 \%$ \\
\hline Squash & 157 & $7 \%$ & 27 & 156 & $487 \%$ & $1 \%$ \\
\hline Top 5 Total & 983 & $42 \%$ & 589 & 965 & $67 \%$ & $2 \%$ \\
\hline Mexico Total & 2,348 & $100 \%$ & 1,740 & 2,232 & $35 \%$ & $5 \%$ \\
\hline
\end{tabular}

Source: AMS Transportation Services Division analysis of AMS Specialty Crops Program Market News data.

"-" indicates no reported shipments during the quarter.

Note: Table values may not conform exactly due to rounding. 
Table 14: Top 5 Commodities Shipped to U.S. from Mexico by State of Entry (1,000 tons)

\begin{tabular}{|c|c|c|c|c|c|c|c|}
\hline \multicolumn{2}{|l|}{ Texas } & \multicolumn{2}{|c|}{ California } & \multicolumn{2}{|l|}{ Arizona } & \multicolumn{2}{|c|}{ New Mexico } \\
\hline Commodity & $\begin{array}{c}\text { 4th } \\
\text { Quarter } \\
2019 \\
\end{array}$ & Commodity & $\begin{array}{c}\text { 4th } \\
\text { Quarter } \\
2019 \\
\end{array}$ & Commodity & $\begin{array}{c}\text { 4th } \\
\text { Quarter } \\
2019 \\
\end{array}$ & Commodity & $\begin{array}{c}\text { 4th } \\
\text { Quarter } \\
2019 \\
\end{array}$ \\
\hline Avocados & 286.24 & $\begin{array}{c}\text { Tomatoes, Plum } \\
\text { Type }\end{array}$ & 48.76 & Cucumbers & 142.54 & Peppers, Other & 85.77 \\
\hline Limes & 128.79 & Onions Green & 39.63 & Squash & 140.22 & Corn-Sweet & 0.38 \\
\hline Tomatoes & 123.16 & Misc Tropical & 29.24 & $\begin{array}{l}\text { Watermelons, } \\
\text { Seedless }\end{array}$ & 105.33 & Misc Tropical & 0.36 \\
\hline $\begin{array}{l}\text { Tomatoes, } \\
\text { Plum Type }\end{array}$ & 74.32 & Asparagus & 20.12 & $\begin{array}{l}\text { Peppers, Bell } \\
\text { Type }\end{array}$ & 60.00 & Watermelons & 0.08 \\
\hline Broccoli & 67.07 & Cucumbers & 15.64 & Tomatoes & 30.23 & $\begin{array}{l}\text { Mixed And } \\
\text { Misc Melons }\end{array}$ & 0.02 \\
\hline $\begin{array}{c}\text { Mexico through } \\
\text { TX Total }\end{array}$ & 1253.10 & $\begin{array}{c}\text { Mexico through } \\
\text { CA Total }\end{array}$ & 327.34 & $\begin{array}{c}\text { Mexico through } \\
\text { AZ Total }\end{array}$ & 680.88 & $\begin{array}{c}\text { Mexico through } \\
\text { NM Total }\end{array}$ & 86.62 \\
\hline
\end{tabular}

Source: AMS Transportation Services Division analysis of AMS Specialty Crops Program Market News data.

"-" indicates no reported shipments during the quarter.

Note: Table values may not conform exactly due to rounding.

Figure 7: Truck Rates from U.S.-Mexico Border (\$/Mile by Distance Traveled)

$\$ 4.00$

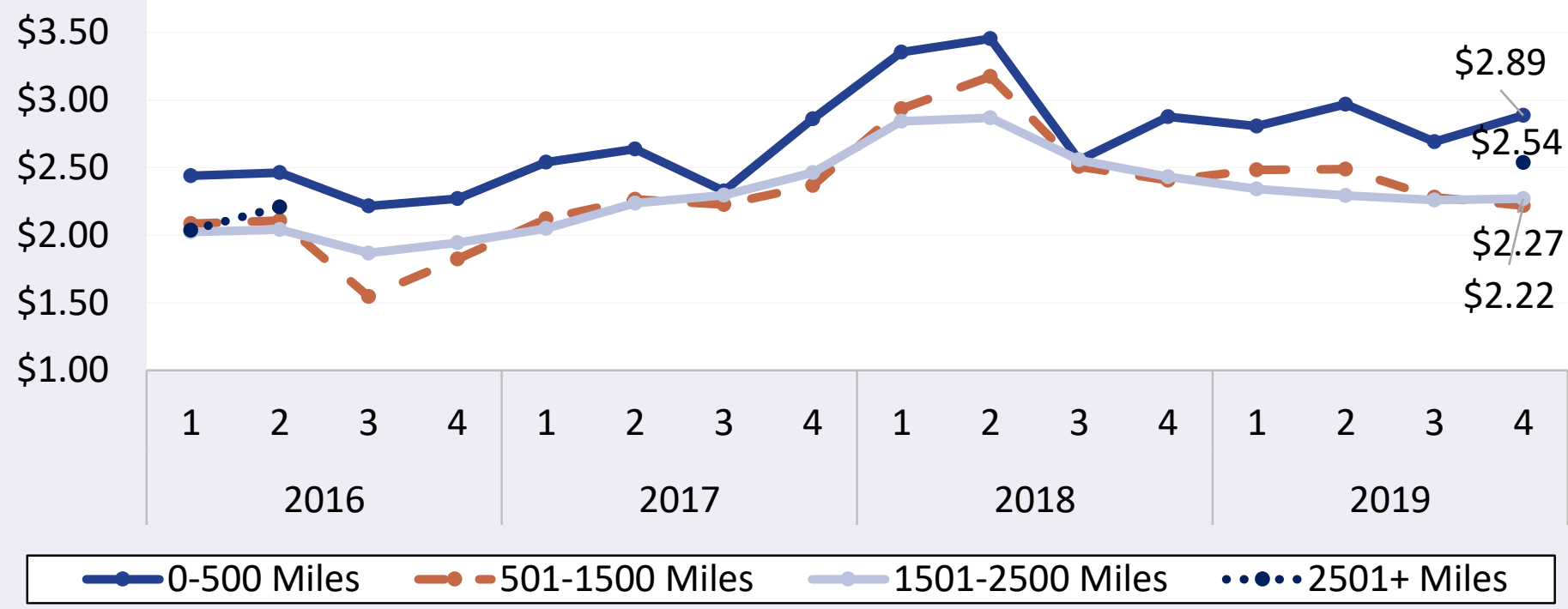

Source: AMS Transportation Services Division analysis of AMS Specialty Crops Program Market News data.

Gaps in the chart lines are the result of quarters with no reported data for the region. 


\section{Agricultural Refrigerated Truck Quarterly \\ Quarter 4, 2019}

Figure 8: Texas Truck Rates from U.S.-Mexico Border (\$/Mile by Distance Traveled) $\$ 4.00$

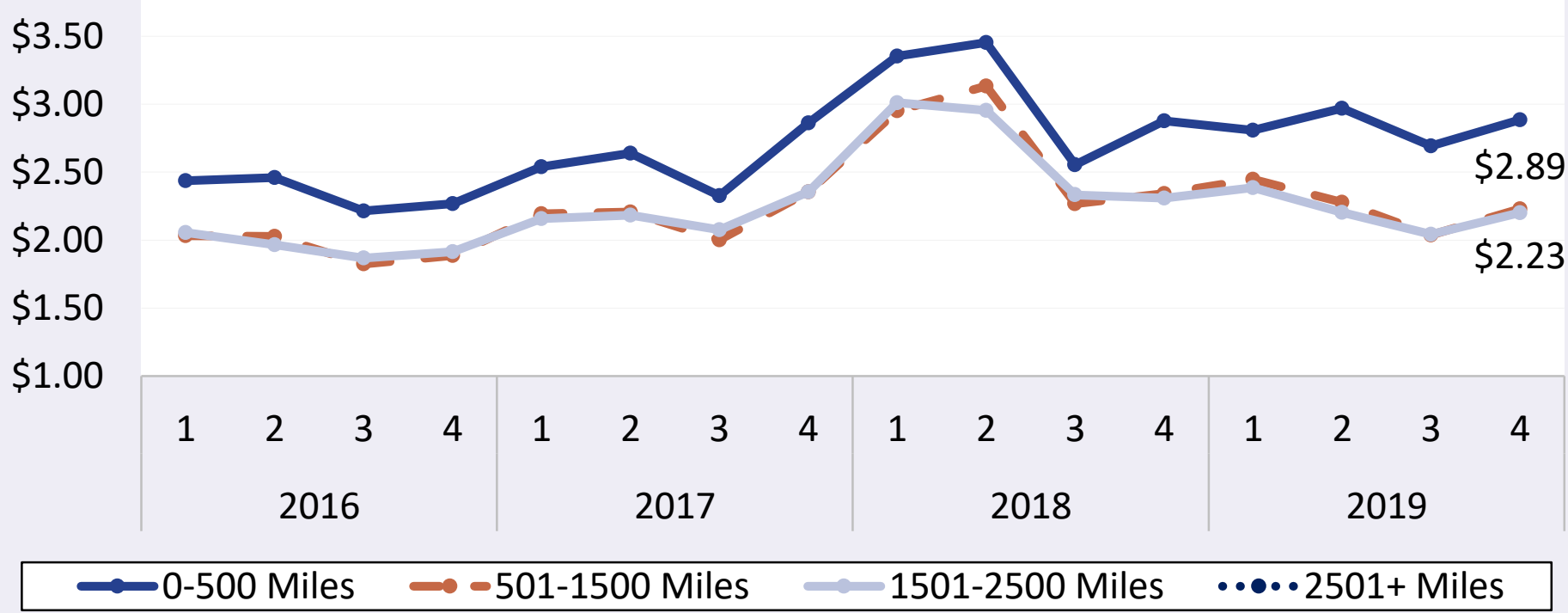

Source: AMS Transportation Services Division analysis of AMS Specialty Crops Program Market News data.

Gaps in the chart lines are the result of quarters with no reported data for the region.

Figure 9: Arizona Truck Rates from U.S.-Mexico Border ( $\$ /$ Mile by Distance Traveled)

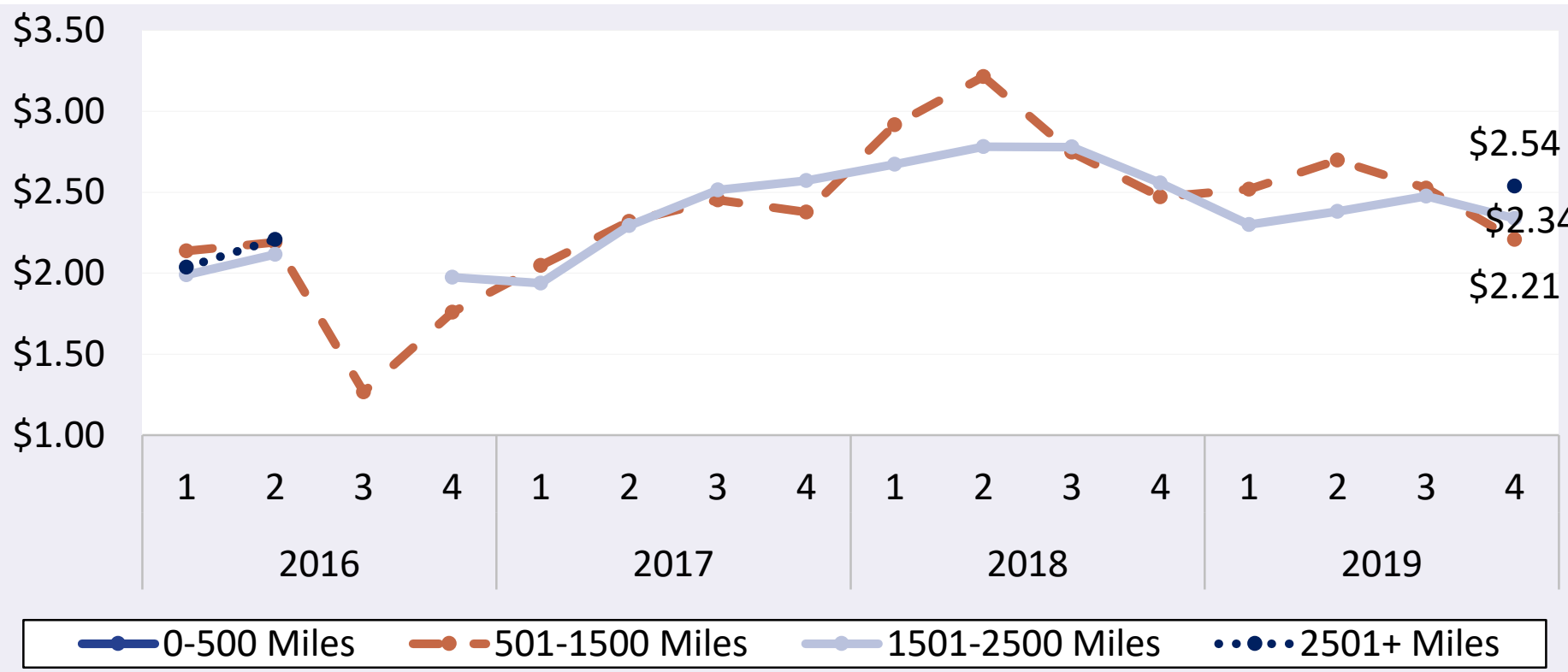

Source: AMS Transportation Services Division analysis of AMS Specialty Crops Program Market News data.

Gaps in the chart lines are the result of quarters with no reported data for the region. 
Quarter 4, 2019

Table 15: Truck Overview from the U.S.-Mexico Border

\begin{tabular}{|r|c|c|c|c|}
\hline \multirow{2}{*}{ Region/Reporting District } & \multicolumn{4}{|c|}{ Availability Rating, 1=Surplus to 5=Shortage } \\
\cline { 2 - 5 } & October & November & December & 4th Quarter \\
\hline Mexico Crossings Through Nogales, Arizona & 3 & 3.57 & 3.65 & 3.41 \\
\hline Mexico Crossings Through Texas & 3 & 3 & 3 & 3 \\
\hline Regional Average Availability & 3 & 3.29 & 3.33 & 3.20 \\
\hline Diesel Fuel Price (\$/gallon), through Texas & $\mathbf{2 . 8 0}$ & $\mathbf{2 . 7 9}$ & $\mathbf{2 . 7 7}$ & $\mathbf{2 . 7 9}$ \\
\hline Diesel Fuel Price (\$/gallon), through Arizona & $\mathbf{3 . 2 8}$ & $\mathbf{3 . 4 2}$ & $\mathbf{3 . 3 0}$ & $\mathbf{3 . 3 3}$ \\
\hline
\end{tabular}

Source: AMS Transportation Services Division analysis of AMS Specialty Crops Program Market News data.

"-" indicates no reported shipments during the quarter.

Note: Table values may not conform exactly due to rounding.

\section{Figure 10: Refrigerated Truck Availability Monthly Ratings from the U.S-Mexico Border}

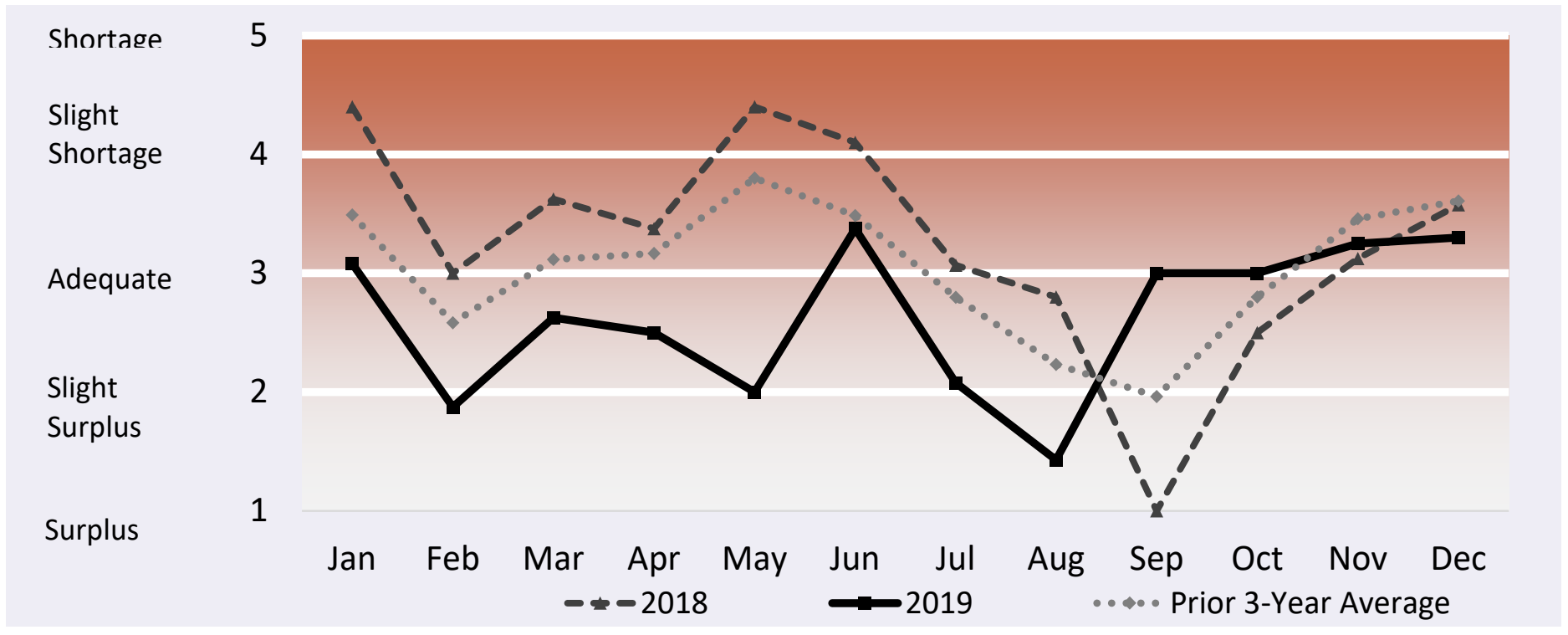

Source: AMS Transportation Services Division analysis of AMS Specialty Crops Program Market News data.

Gaps in the chart lines are the result of quarters with no reported data for the region. 


\section{PNW}

\section{Volume}

During fourth quarter 2019, total reported shipments of fruits and vegetables from the Pacific Northwest were 1.87 million tons, increasing 3 percent year to year. The sum of the top five commodities also increased 3 percent year to year. Year-to-year increases in apple (17 percent) and cranberry ( 5 percent) shipments were offset by decreases in potato ( 2 percent), dry onion ( 7 percent), and pear ( 1 percent) shipments.

\section{Rates}

The quarterly average truck rate for shipments between 501 miles and 1,500 miles was $\$ 2.18$ per mile, increasing 14 percent quarter to quarter, but decreasing 11 percent year to year.

\section{Truck Overview}

Diesel fuel prices averaged $\$ 3.33$ per gallon, increasing 5 percent quarter to quarter but decreasing 4 percent year to year. Shippers reported adequate truck availability through mid-November followed by slight shortage and shortage conditions through the end of 2019.

Table 16: Reported Top Five Commodities Shipped from the PNW (1,000 tons)

\begin{tabular}{|c|c|c|c|c|c|c|}
\hline \multirow{2}{*}{ Commodity } & \multirow{2}{*}{$\begin{array}{c}\text { 4th Quarter } \\
2019\end{array}$} & \multirow{2}{*}{$\begin{array}{l}\text { Share of } \\
\text { PNW Total }\end{array}$} & \multirow{2}{*}{$\begin{array}{l}\text { Previous } \\
\text { Quarter }\end{array}$} & \multirow{2}{*}{$\begin{array}{c}\text { Same } \\
\text { Quarter Last } \\
\text { Year }\end{array}$} & \multicolumn{2}{|c|}{$\begin{array}{l}\text { Current Quarter as \% } \\
\text { change from: }\end{array}$} \\
\hline & & & & & Previous Qtr & $\begin{array}{l}\text { Same Qtr } \\
\text { Last Year }\end{array}$ \\
\hline Apples & 741 & $40 \%$ & 558 & 635 & $33 \%$ & $17 \%$ \\
\hline Potatoes & 531 & $28 \%$ & 547 & 542 & $-3 \%$ & $-2 \%$ \\
\hline Onions Dry & 431 & $23 \%$ & 242 & 463 & $78 \%$ & $-7 \%$ \\
\hline Pears & 165 & $9 \%$ & 52 & 167 & $220 \%$ & $-1 \%$ \\
\hline Cranberries & 2 & $0 \%$ & 0 & 2 & $4,538 \%$ & $5 \%$ \\
\hline Top 5 Total & 1,871 & $100 \%$ & 1,399 & 1,809 & $34 \%$ & $3 \%$ \\
\hline PNW Total & 1,871 & $100 \%$ & 1,551 & 1,809 & $21 \%$ & $3 \%$ \\
\hline
\end{tabular}

Source: AMS Transportation Services Division analysis of AMS Specialty Crops Program Market News data.

"-" indicates no reported shipments during the quarter.

Note: Table values may not conform exactly due to rounding. 
Figure 11: PNW Truck Rates (\$/Mile by Distance Traveled)

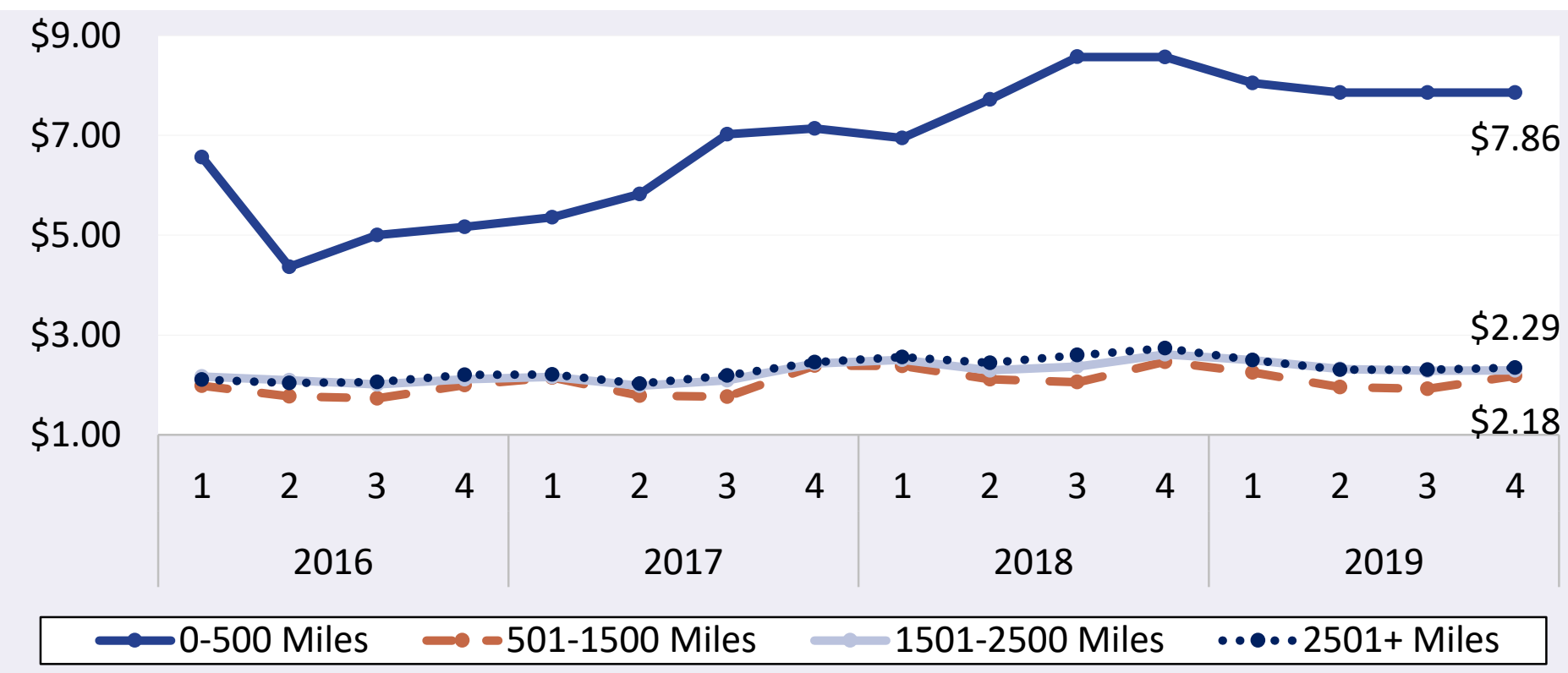

Source: AMS Transportation Services Division analysis of AMS Specialty Crops Program Market News data.

Gaps in the chart lines are the result of quarters with no reported data for the region.

Table 17: PNW Truck Overview (Availability Rating: 1=Surplus to 5=Shortage)

\begin{tabular}{|c|c|c|c|c|}
\hline \multirow{2}{*}{ Region/Reporting District } & \multicolumn{3}{|c|}{ Availability Rating, 1=Surplus to 5=Shortage } \\
\cline { 2 - 5 } & October & November & December & 4th Quarter \\
\hline Columbia Basin Washington & 3 & 3.56 & 4.17 & 3.57 \\
\hline Idaho And Malheur County, Oregon & 3 & 3.5 & 3.87 & 3.46 \\
\hline Upper Valley, Twin Falls-Burley District Idaho & 3.2 & 3.5 & 4 & 3.57 \\
\hline Yakima Valley \& Wenatchee District Washington & 3 & 3.25 & 3.4 & 3.22 \\
\hline Regional Average Availability & 3.05 & 3.45 & 3.86 & 3.45 \\
\hline Diesel Fuel Price (\$/gallon) & 3.28 & 3.42 & 3.30 & 3.33 \\
\hline
\end{tabular}

Source: AMS Transportation Services Division analysis of AMS Specialty Crops Program Market News data.

"-" indicates no reported shipments during the quarter.

Note: Table values may not conform exactly due to rounding. 


\section{Agricultural Refrigerated Truck Quarterly \\ Quarter 4, 2019}

Figure 12: Refrigerated Truck Availability Monthly Ratings for the PNW

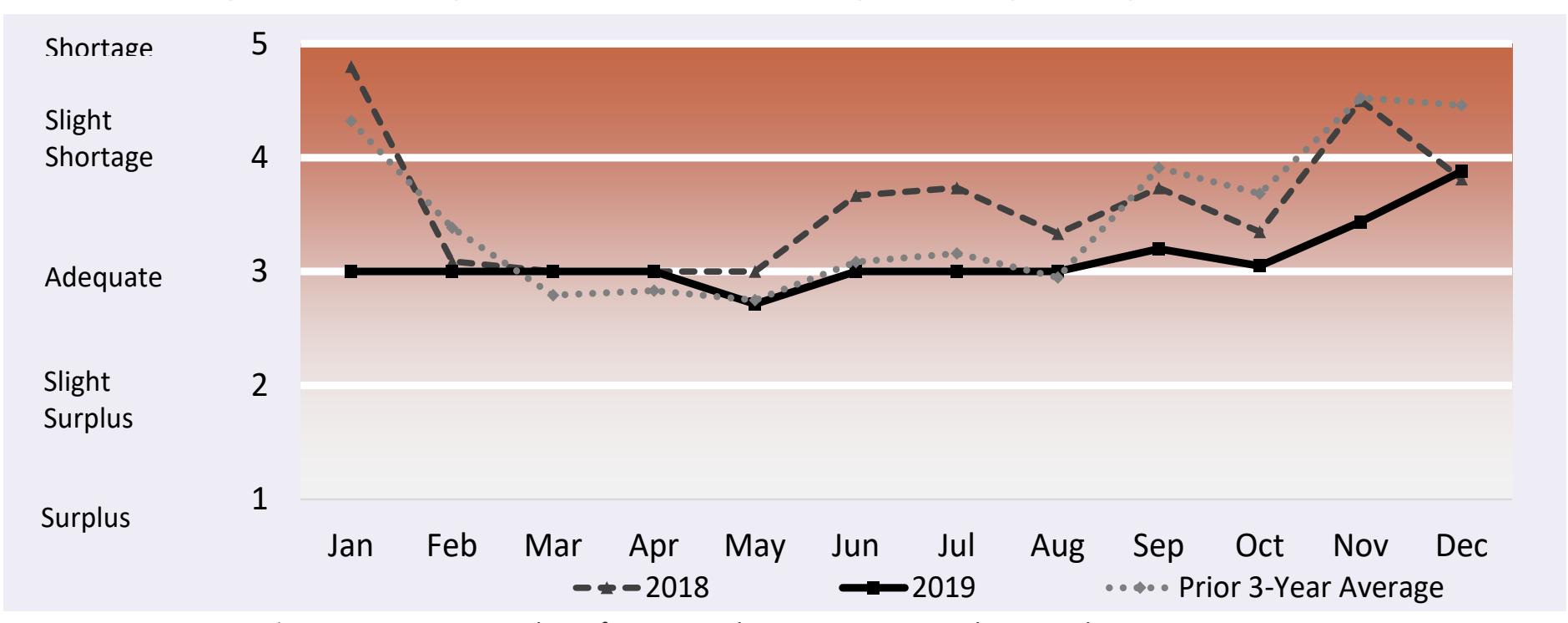

Source: AMS Transportation Services Division analysis of AMS Specialty Crops Program Market News data.

Gaps in the chart lines are the result of quarters with no reported data for the region. 


\section{Arizona}

\section{Volume}

During fourth quarter 2019, total reported shipments of fruits and vegetables from Arizona were 422,000 tons, down 12 percent year to year. The sum of the top five commodities also decreased 12 percent quarter to quarter. Shipments of each of the top five commodities decreased including relatively significant decreases in iceberg lettuce (14 percent), processed lettuce (18 percent), and cantaloupe (24 percent).

\section{Rates}

The quarterly average truck rate for shipments between 501 miles and 1,500 miles was \$2.98 per mile, down 2 percent quarter to quarter, and down 26 percent year to year.

\section{Truck Overview}

Diesel fuel prices averaged $\$ 3.33$ per gallon, up 5 percent quarter to quarter but down 4 percent year to year. Shippers reported adequate truck availability for most of fourth quarter 2019 except for slight shortage conditions the last week in November and shortage conditions the last 2 weeks in December.

Table 18: Reported Top Five Commodities Shipped from Arizona (1,000 tons)

\begin{tabular}{|c|c|c|c|c|c|c|}
\hline \multirow{2}{*}{ Commodity } & \multirow{2}{*}{$\begin{array}{c}\text { 4th Quarter } \\
2019\end{array}$} & \multirow{2}{*}{$\begin{array}{c}\text { Share of } \\
\text { Arizona Total }\end{array}$} & \multirow{2}{*}{$\begin{array}{l}\text { Previous } \\
\text { Quarter }\end{array}$} & \multirow{2}{*}{$\begin{array}{c}\text { Same } \\
\text { Quarter Last } \\
\text { Year }\end{array}$} & \multicolumn{2}{|c|}{$\begin{array}{l}\text { Current Quarter as \% } \\
\text { change from: }\end{array}$} \\
\hline & & & & & Previous Qtr & $\begin{array}{l}\text { Same Qtr } \\
\text { Last Year }\end{array}$ \\
\hline Lettuce, Iceberg & 128 & $30 \%$ & 0 & 149 & - & $-14 \%$ \\
\hline Lettuce, Romaine & 114 & $27 \%$ & 0 & 119 & - & $-4 \%$ \\
\hline Lettuce, Processed & 55 & $13 \%$ & 0 & 67 & - & $-18 \%$ \\
\hline Cantaloupe & 27 & $6 \%$ & 16 & 36 & $68 \%$ & $-24 \%$ \\
\hline Lettuce, Green Leaf & 18 & $4 \%$ & 0 & 18 & - & $-1 \%$ \\
\hline Top 5 Total & 342 & $81 \%$ & 16 & 388 & $2,001 \%$ & $-12 \%$ \\
\hline Arizona Total & 422 & $100 \%$ & 34 & 479 & $1129 \%$ & $-12 \%$ \\
\hline
\end{tabular}

Source: AMS Transportation Services Division analysis of AMS Specialty Crops Program Market News data.

"_" indicates no reported shipments during the quarter.

Note: Table values may not conform exactly due to rounding. 
Figure 13: Arizona Truck Rates (\$/Mile by Distance Traveled)

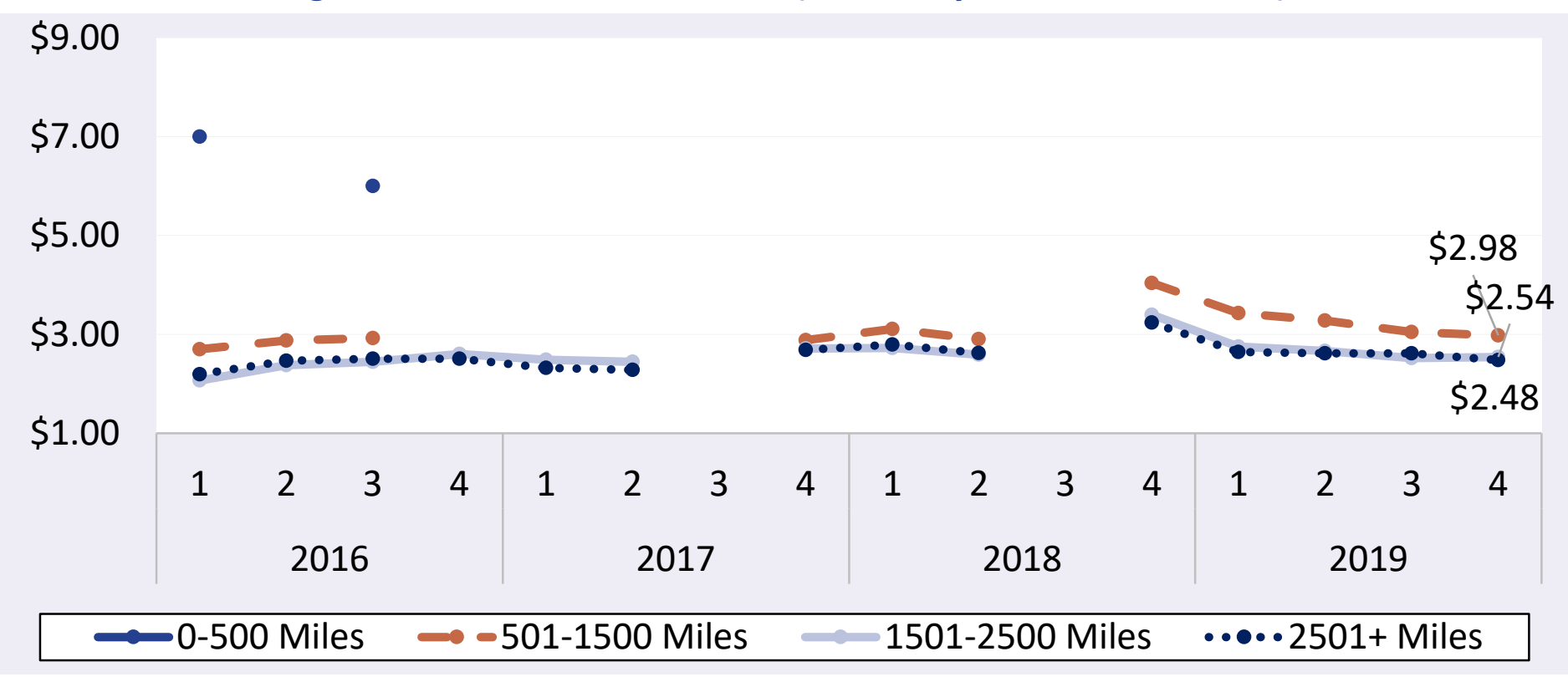

Source: AMS Transportation Services Division analysis of AMS Specialty Crops Program Market News data.

Gaps in the chart lines are the result of quarters with no reported data for the region.

Table 19: Arizona Truck Overview (Availability Rating: 1=Surplus to 5=Shortage)

\begin{tabular}{|r|c|c|c|c|}
\hline Region/Reporting District & October & November & December & 4th Quarter \\
\hline Central And Western Arizona & 3 & 3.33 & - & 3.17 \\
\hline $\begin{array}{r}\text { Imperial, Palo Verde And Coachella Valleys, } \\
\text { California And Central And Western Arizona }\end{array}$ & - & - & 3.6 & 3.6 \\
\hline Regional Average Availability & 3 & 3.33 & 3.6 & 3.31 \\
\hline Diesel Fuel Price (\$/gallon) & 3.28 & 3.42 & 3.30 & 3.33 \\
\hline
\end{tabular}

Source: AMS Transportation Services Division analysis of AMS Specialty Crops Program Market News data.

"_" indicates no reported shipments during the quarter.

Note: Table values may not conform exactly due to rounding. 


\section{Agricultural Refrigerated Truck Quarterly \\ Quarter 4, 2019}

Figure 14: Refrigerated Truck Availability Monthly Ratings for Arizona

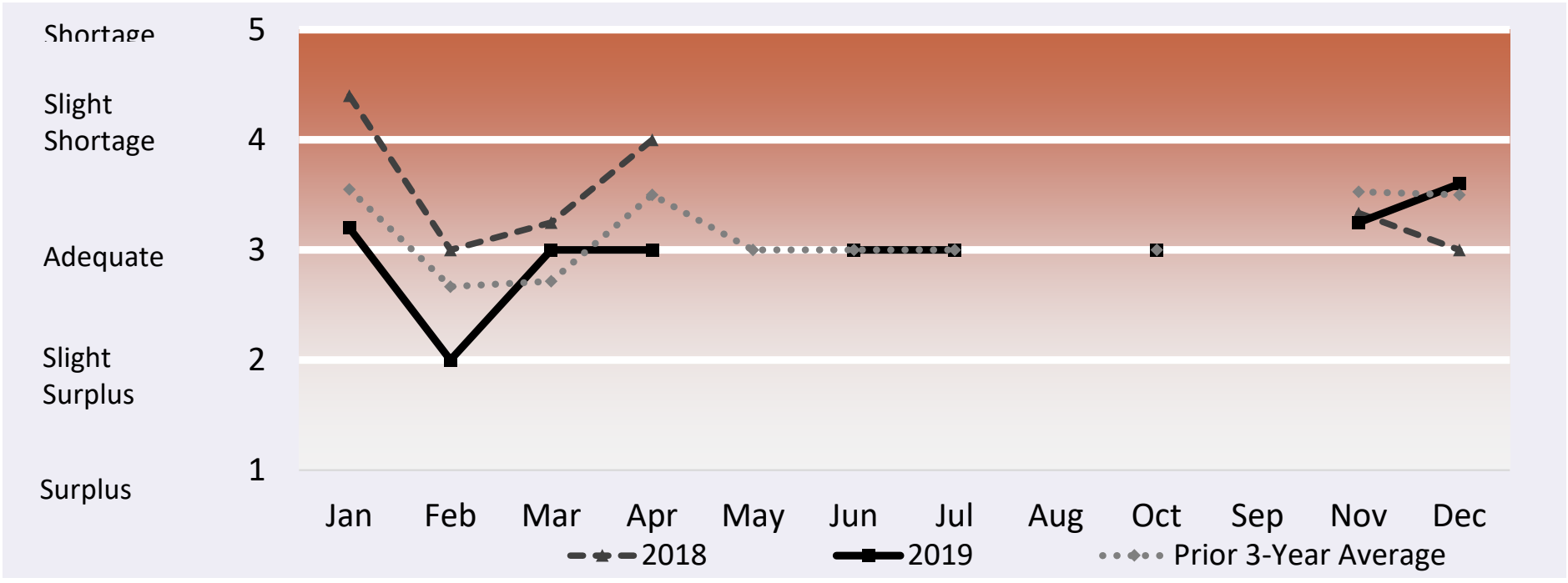

Source: AMS Transportation Services Division analysis of AMS Specialty Crops Program Market News data.

Gaps in the chart lines are the result of quarters with no reported data for the region. 


\section{Florida}

\section{Volume}

Total reported shipments of fruits and vegetables from Florida during fourth quarter 2019 were 387,000 tons, up 4 percent year to year. The sum of the top five commodities increased 2 percent year to year. Three of the top five commodities-including oranges (up 21 percent), grapefruit (up 2 percent), and bell peppers (up 24 percent)-increased year to year. However, shipments of tomatoes (down 3 percent) and plum tomatoes (down 13 percent) decreased year to year.

\section{Rates}

The quarterly average truck rate for shipments between 501 miles and 1,500 miles was $\$ 1.90$ per mile, down 32 percent quarter to quarter and down 12 percent year to year.

\section{Truck Overview}

Diesel fuel prices averaged \$2.92 per gallon, up 0.3 percent quarter to quarter but down 7 percent year to year. Shippers reported adequate and slight surplus truck availability until the end of December when shippers reported slight shortage and shortage conditions.

Table 20: Reported Top Five Commodities Shipped from Florida (1,000 tons)

\begin{tabular}{|c|c|c|c|c|c|c|}
\hline \multirow{2}{*}{ Commodity } & \multirow{2}{*}{$\begin{array}{c}\text { 4th Quarter } \\
2019\end{array}$} & \multirow{2}{*}{$\begin{array}{l}\text { Share of } \\
\text { Florida Total }\end{array}$} & \multirow{2}{*}{$\begin{array}{c}\text { Previous } \\
\text { Quarter }\end{array}$} & \multirow{2}{*}{$\begin{array}{c}\text { Same } \\
\text { Quarter Last } \\
\text { Year }\end{array}$} & \multicolumn{2}{|c|}{$\begin{array}{l}\text { Current Quarter as \% } \\
\text { change from: }\end{array}$} \\
\hline & & & & & Previous Qtr & $\begin{array}{l}\text { Same Qtr } \\
\text { Last Year }\end{array}$ \\
\hline Tomatoes & 106 & $28 \%$ & 0 & 110 & - & $-3 \%$ \\
\hline Oranges & 38 & $10 \%$ & 2 & 31 & $2,339 \%$ & $21 \%$ \\
\hline Grapefruit & 34 & $9 \%$ & 0 & 34 & - & $2 \%$ \\
\hline Tomatoes, Plum Type & 29 & $8 \%$ & - & 34 & - & $-13 \%$ \\
\hline Peppers, Bell Type & 24 & $6 \%$ & 0 & 19 & - & $24 \%$ \\
\hline Top 5 Total & 232 & $60 \%$ & 2 & 228 & - & $2 \%$ \\
\hline Florida Total & 387 & $100 \%$ & 21 & 371 & $1710 \%$ & $4 \%$ \\
\hline
\end{tabular}

Source: AMS Transportation Services Division analysis of AMS Specialty Crops Program Market News data.

"_" indicates no reported shipments during the quarter.

Note: Table values may not conform exactly due to rounding. 


\section{Agricultural Refrigerated Truck Quarterly \\ Quarter 4, 2019}

Figure 15: Florida Truck Rates (\$/Mile by Distance Traveled)

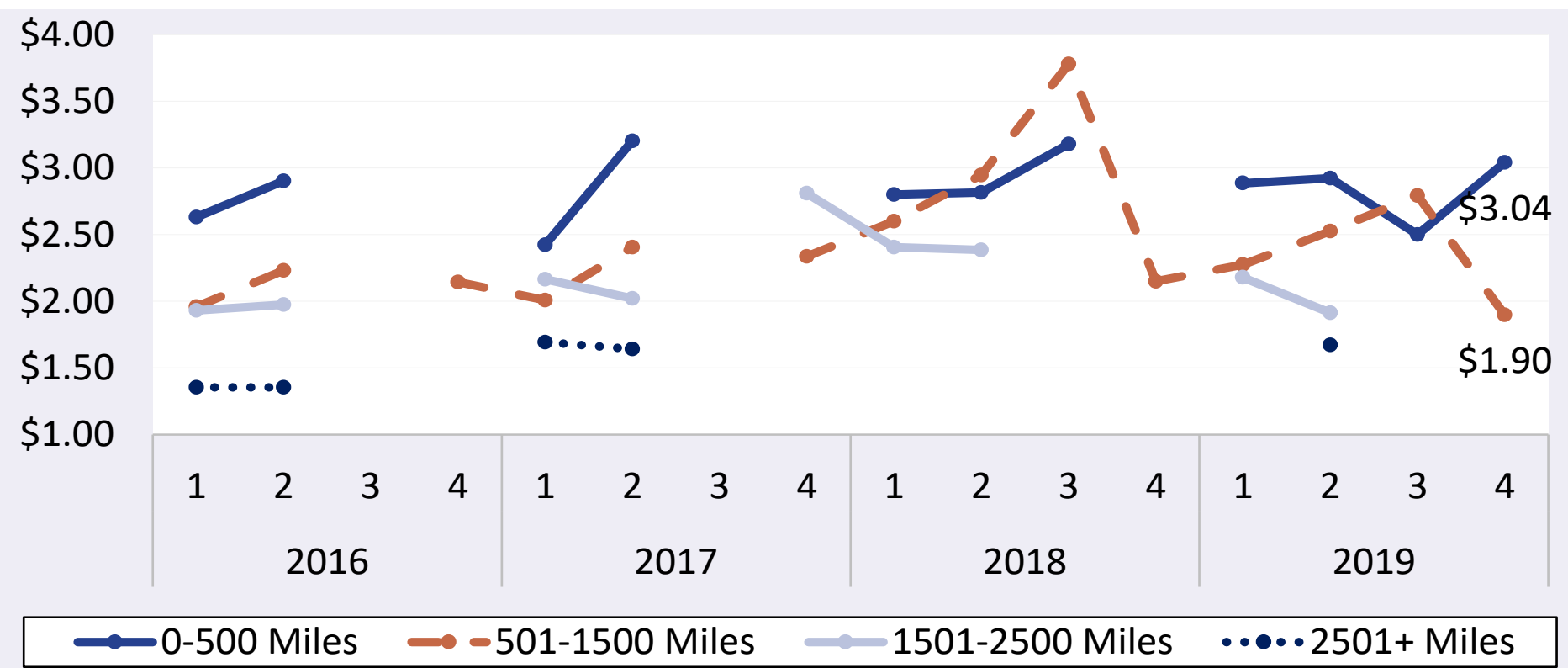

Source: AMS Transportation Services Division analysis of AMS Specialty Crops Program Market News data.

Gaps in the chart lines are the result of quarters with no reported data for the region.

Table 21: Florida Truck Overview (Availability Rating: 1=Surplus to 5=Shortage)

\begin{tabular}{|r|c|c|c|c|}
\hline \multirow{2}{*}{ Region/Reporting District } & \multicolumn{4}{|c|}{ Availability Rating, 1=Surplus to 5=Shortage } \\
\cline { 2 - 5 } & October & November & December & 4th Quarter \\
\hline Central \& South Florida & - & 2.5 & 4 & 3.25 \\
\hline West District Florida & 3 & 2.5 & - & 2.75 \\
\hline Regional Average Availability & 3 & 2.5 & 4 & 3.17 \\
\hline Diesel Fuel Price (\$/gallon) & $\mathbf{2 . 9 2}$ & $\mathbf{2 . 9 2}$ & $\mathbf{2 . 9 3}$ & $\mathbf{2 . 9 2}$ \\
\hline
\end{tabular}

Source: AMS Transportation Services Division analysis of AMS Specialty Crops Program Market News data.

"-" indicates no reported shipments during the quarter.

Note: Table values may not conform exactly due to rounding. 


\section{Agricultural Refrigerated Truck Quarterly \\ Quarter 4, 2019}

Figure 16: Refrigerated Truck Availability Monthly Ratings for Florida

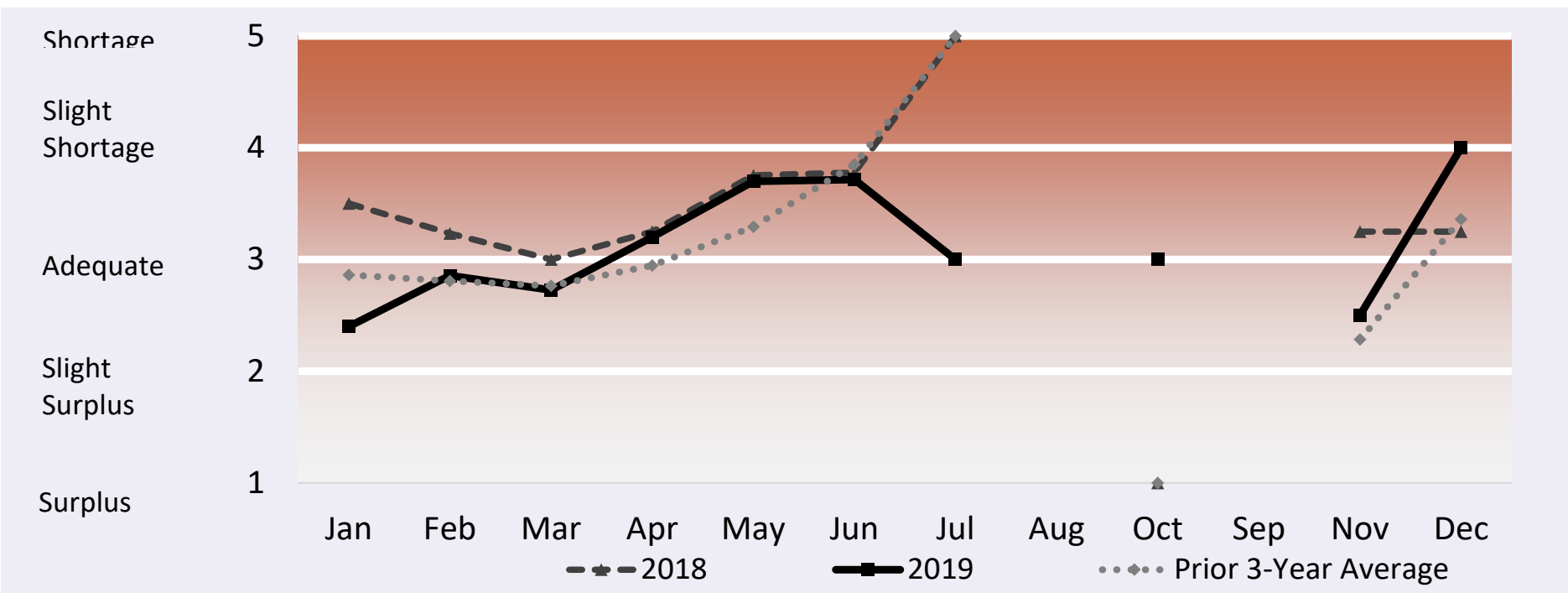

Source: AMS Transportation Services Division analysis of AMS Specialty Crops Program Market News data.

Gaps in the chart lines are the result of quarters with no reported data for the region. 


\section{TERMS AND REFERENCES}

\section{Data Sources}

This information is compiled from the weekly Specialty Crops Truck Rate Report by USDA, Agricultural Marketing Service (AMS), Specialty Crops Program, Market News Division. The website is: https://www.marketnews.usda.gov/mnp/fv-home.

\section{Regional Markets}

For the regional markets, some States are grouped into producing regions. The Pacific Northwest region includes Idaho, Oregon, and Washington. The Great Lakes region includes Michigan, Minnesota, and Wisconsin. The Southeast region includes North Carolina, South Carolina and Georgia.

\section{Shipment Volumes}

Truck shipments for all commodities and origins are not available. Those obtainable are reported, but should not be interpreted as representing complete movements of a commodity. Truck shipments from all States are collected at shipping points and include both interstate and intrastate movements. They are obtained from various sources, including Federal marketing orders, administrative committees, Federal State Inspection Service, and shippers. Volume amounts are represented in 10,000 pound units, or 1,000 10-lb packages but are converted to 1,000 tons for this report. Mexican border crossings through Arizona and Texas data is obtained from the Department of Homeland Security (DHS), U.S. Customs and Border and Protection (CBP) through USDA, AMS, Market News.

\section{Rates}

This information is compiled from the weekly Specialty Crops Truck Rate Report. Rates quoted represent open (spot) market rates that shippers or receivers pay depending on basis of sale, per load, including truck brokers fees for shipments in truck load volume to a single destination. Extra charges for delivery to terminal markets, multipickup and multidrop shipments are not included unless otherwise stated. Rates are based on the most usual loads in 48-53 foot trailers from the origin shipping area to the destination receiving city. In areas where rates are based on package rates, per load rates were derived by multiplying the package rate by the number of packages in the most usual load in a 48-53 foot trailer. Slightly cheaper rates will be reported during Quarters 2 and 3 as about 50 percent of onion shipments from California are hauled on open flatbed trailers. During Quarter 3, less than 20 percent of onions hauled from Washington, Idaho, and Oregon are on open flatbeds.

\section{Regional Rates}

Rate data for 10 destination markets are used to calculate average origin regional rates.

\section{National Rates}

The national rates reflect the average of the regional rates, separated by mileage category and weighted by volume between origin and destination. 


\section{CONTACT INFORMATION}

Authors

April Taylor, Coordinator; Quarterly Overview and U.S. Diesel Prices

April.Taylor@usda.gov, 202.720.7880

Brian McGregor, Regulatory News/Updates

Brian.McGregor@usda.gov, 202.720.0035

Jesse Gastelle, Data Analysis

Jesse.Gastelle@usda.gov, 202.690.1144

Patty Willkie, Specialty Crops Program and Market News Division Data

Patty.Willkie@usda.gov, 202.720.2175

To subscribe, please send an e-mail to April.Taylor@usda.gov. Printed copies are available upon request.

For assistance with accessibility issues related to this document, please e-mail SharonC.Williams@usda.gov.

Related Websites

USDA's Agricultural Transportation Open Data Platform

https://agtransport.usda.gov/

Specialty Crops Program

http://www.ams.usda.gov/about-ams/programs-offices/specialty-crops-program

Specialty Crops Truck Rate Report

http://www.ams.usda.gov/market-news/fruits-vegetables

Economic Research Service Vegetable and Pulses

https://www.ers.usda.gov/topics/crops/vegetables-pulses/

Economic Research Service Fruit and Tree Nuts

http://www.ers.usda.gov/topics/crops/fruit-tree-nuts.aspx

National Agricultural Statistics Service, Crops

http://www.nass.usda.gov/Statistics by Subject/index.php?sector=CROPS

Refrigerated Truck Quarterly Datasets

https://www.ams.usda.gov/services/transportation-analysis/agricultural-refrigerated-truck-quarterly-datasets

Protecting Perishable Foods During Transport by Truck and Rail

https://edis.ifas.ufl.edu/pdffiles/HS/HS132800.pdf

\section{Preferred Citation}

U.S. Department of Agriculture, Agricultural Marketing Service. Agricultural Refrigerated Truck Quarterly Report. July 2020. Web. <https://dx.doi.org/10.9752/TS051.07-2020>

\section{Photo Credit}

Burt Barnes

USDA is an equal opportunity provider, employer, and lender. 